\title{
13. GRAIN SIZE AND MINERALOGY OF SEDIMENTS AND SEDIMENTARY ROCKS, DSDP LEG 35'
}

\author{
Yu. A. Bogdanov, V.N. Zhivago, A.P. Lisitzin, V.V. Serova, and E.N. Zharikova, \\ P.P. Shirshov Institute of Oceanology, Academy of Sciences, USSR
}

\section{INTRODUCTION}

The grain size and mineralogy of sediments and sedimentary rocks drilled during DSDP Leg 35 have been influenced by several factors, including the tectonic development of the adjacent land (primarily the coast of West Antarctica), the evolution of surface and bottom circulation, glaciation on Antarctica, and changes in biogenic productivity.

We conducted the grain-size and mineralogical studies in accordance with the standard method adopted by the P.P. Shirshov Institute of Oceanology, Academy of Sciences, USSR; the techniques are described by A.P. Lisitzin and others in the Initial Reports of the Deep Sea Drilling Project, Volume 6.

\section{GRAIN SIZE}

The results of the mechanical grain-size analyses, utilizing the combined method (Lisitzin et al., 1969), are given in Tables 1-4. Grain-size analyses for fractions of 0.05 to $0.1 \mathrm{~mm}$ were also made on the light and heavy fractions obtained by heavy liquid $\left(2.9 \mathrm{~g} / \mathrm{cm}^{3}\right)$ separation. The mineralogy of the coarse aleuritic fractions obtained by heavy liquid separation is presented in Tables 5-10. We conducted a statistical study of the distribution of individual fractions and investigated significant textural characteristics of the sediments, such as the median grain diameter $(M d)$ and the sorting coefficient $(\mathrm{So})$. From these studies of distribution of the $M d$ and $S o$ values, we present this preliminary discussion of the grain size of sediments from the Southern Ocean drilled during DSDP Leg 35.

\section{Site 322}

The sedimentary strata can be subdivided into three lithologic units at Site 322.

Unit 1 comprises approximately 300 meters of upper Miocene and Pliocene, interbedded, slightly consolidated to unconsolidated sands, aleurites, and pelites. The grain-size distribution of sediments is extremely heterogeneous throughout the unit. In the upper part, the individual interlayers of sands and aleurites are well sorted ( $S o$ reaches 1.93) whereas lower in the unit, the sorting, as interpreted from the cumulative curves, is much poorer. Most of the interlayers, and especially those in the lower part of the unit are characterized by a high pelite content (up to $45 \%-55 \%$ ). The values of $M d$, which reach $0.035 \mathrm{~mm}$ in the upper part, fluctuate at the base of the unit between 0.0015 and $0.0020 \mathrm{~mm}$.

'Translated from Russian.
Unit 2 consists of 171 meters of middle to upper Miocene clays. This unit is the extension of the lower part of Unit 1, but differs in being homogeneous. The sediment here is primarily pelite, of which more than $40 \%$ is less than $0.001 \mathrm{~mm}$. The values of $M d$ vary within the same range as those in Unit 1 (lower part), and sediments are well sorted in most layers as indicated by the slope of the cumulative curves.

Unit 3 (=Units 3 and 4 in the Site Report chapters, this volume), approximately 47 meters of middle or possibly lower Miocene sediment, differs rather distinctly from the overlying unit and is characterized by the intercalation of consolidated sandstones and aleurites. The sediments are generally rather poorly sorted (So fluctuates from 2.3 to 5.0) and contain substantially less pelitic material than Unit 2 (up to $20 \%$ $25 \%)$.

\section{Site 323}

Texture and other lithologic parameters were studied in detail from the sediments of Site 323 and enabled us to define four units.

Unit 1, the thickest of this sequence, comprises 266 meters of Pliocene and Miocene intercalated pelites and aleurites with admixtures of sand-sized organic remains in some parts of the section. Sorting is rather poor; values of So vary from 2.8 to 4.8 . Pelitic material predominates (70\%-90\%) throughout the entire interval. In the upper part of the unit, the values of $M d$ vary quite markedly from 0.001 to $0.12 \mathrm{~mm}$. However, in the lowermost strata, the values of $M d$ do not exceed 0.003$0.004 \mathrm{~mm}$. Overall, this unit is both lithologically similar and stratigraphically equivalent to Unit 1 from Site 322.

Unit 2 consists of 240 meters of middle to lower Miocene consolidated pelitic sediments, which contain an admixture of silica and a large proportion of radiolarian and diatom remains. A characteristic feature of the unit is the higher degree of sorting with increased depth (So ranges from 3.66 to 1.44). As with the overlying unit, the pelitic fraction dominates, and in many samples constitutes as much as $90 \%-95 \%$ of the sediment. The aleuritic fraction forms a much smaller part and the fine arenaceous fraction, which occurs in the uppermost and lowermost part of Unit 1, is completely absent. The high values of $M d$ in the upper part of Unit $2(0.04-0.05 \mathrm{~mm})$, resulting from interlayers of unconsolidated sands, are replaced down section by $M d$ values of $0.001-0.005 \mathrm{~mm}$.

Unit 3, 130 meters of lower Miocene to ? middle Oligocene consolidated pelitic sediments, differs but little in gross lithology from the overlying Unit 2. However, fine aleuritic materials are noticeably pres- 
TABLE 1

Granulometry of Sediments at Site 322 by the Combined Method

\begin{tabular}{|c|c|c|c|c|c|c|c|c|c|c|c|c|c|c|}
\hline \multirow{2}{*}{$\begin{array}{c}\text { Sample } \\
\text { (Interval in } \mathrm{cm} \text { ) }\end{array}$} & \multirow{2}{*}{$\begin{array}{l}\text { Weight } \\
\text { Suspension } \\
\text { (g) }\end{array}$} & \multirow{2}{*}{$\begin{array}{c}\% \text { of } \\
\text { Wet Wt. }\end{array}$} & \multicolumn{12}{|c|}{ Weight Percent of Size Fractions (in mm) } \\
\hline & & & $>5$ & $5-3$ & $3-2$ & $2-1$ & $1-0.5$ & $0.5-0.25$ & $0.25-0.1$ & $0.1-0.05$ & $0.05-0.01$ & $0.01-0.005$ & $0.005-0.001$ & $<0.001$ \\
\hline $1-2,26-30$ & 11.26 & 28.2 & - & - & - & - & - & - & 3.91 & 29.40 & 51.77 & 3.82 & 5.06 & 6.04 \\
\hline $1-3,70-76$ & 6.55 & 54.0 & - & - & - & - & - & - & 3.05 & 3.05 & 12.06 & 12.99 & 23.81 & 45.04 \\
\hline $2-2,113-116$ & 8.89 & 43.3 & - & - & - & 2.92 & 7.31 & 3.15 & 0.56 & 0.45 & 0.34 & 8.78 & 21.26 & 55.23 \\
\hline $3-1,18-20$ & 9.53 & 39.4 & - & - & - & - & - & - & 0.21 & 0.21 & 0.84 & 27.60 & 23.92 & 47.22 \\
\hline $4-1,65-67$ & 12.61 & 28.7 & - & - & - & - & - & - & 0.32 & 0.64 & 0.40 & 24.01 & 25.46 & 49.17 \\
\hline $4-2,57-60$ & 11.42 & 26.9 & - & - & - & - & - & - & 1.93 & 9.54 & 4.99 & 11.56 & 33.10 & 38.88 \\
\hline $5-1,81-85$ & 13.39 & 27.1 & - & - & - & - & - & - & 1.12 & 0.45 & 2.91 & 26.66 & 28.23 & 40.63 \\
\hline $6-1,30-33$ & 15.56 & 22.6 & - & - & - & - & - & - & 0.32 & 0.19 & 0.57 & 13.38 & 36.44 & 49.10 \\
\hline $9-2,143-146$ & 16.19 & 22.8 & - & - & - & - & - & - & 1.24 & 14.52 & 58.74 & 5.80 & 8.15 & 11.55 \\
\hline $10-1,101-105$ & 17.15 & 19.17 & - & - & - & 0.70 & 0.29 & 0.82 & 32.24 & 38.02 & 9.04 & 4.88 & 5.21 & 8.80 \\
\hline $10-2,43-46$ & 15.24 & 20.3 & - & - & - & - & 0.13 & 1.31 & 33.66 & 45.01 & 6.89 & 1.12 & 1.58 & 10.30 \\
\hline $11-1,42-46$ & 24.48 & 19.7 & - & - & - & - & 0.16 & 1.23 & 31.78 & 35.54 & 10.29 & 5.02 & 5.93 & 10.05 \\
\hline $11-2,45-49$ & 18.48 & 16.8 & - & - & - & - & 4.60 & 0.81 & 24.57 & 33.01 & 7.36 & 6.66 & 6.65 & 16.34 \\
\hline $11-3,24-32$ & 17.07 & 17.5 & - & - & - & - & 0.12 & 1.58 & 38.37 & 26.83 & 8.90 & 4.34 & 5.57 & 14.29 \\
\hline $11-4,17-24$ & 16.83 & 17.4 & - & - & - & - & 0.12 & 4.58 & 39.63 & 18.18 & 5.76 & 7.84 & 16.87 & 7.02 \\
\hline $11-5,36-40$ & 20.62 & 19.8 & - & - & - & - & - & - & 0.19 & 0.15 & 0.53 & 28.42 & 27.55 & 46.16 \\
\hline $11-6,109-115$ & 10.09 & 22.0 & - & - & - & - & - & - & 0.09 & 0.18 & 1.09 & 19.62 & 19.46 & 59.56 \\
\hline
\end{tabular}

TABLE 2

Granulometry of Sediments at Site 323 by the Combined Method

\begin{tabular}{|c|c|c|c|c|c|c|c|c|c|c|c|c|c|c|}
\hline \multirow{2}{*}{$\begin{array}{c}\text { Sample } \\
\text { (Interval in } \mathrm{cm} \text { ) }\end{array}$} & \multirow{2}{*}{$\begin{array}{l}\text { Weight } \\
\text { Suspension } \\
\text { (g) }\end{array}$} & \multirow{2}{*}{$\begin{array}{c}\% \text { of } \\
\text { Wet Wt. }\end{array}$} & \multirow[b]{2}{*}{$>5$} & \multirow[b]{2}{*}{$5-3$} & \multicolumn{10}{|c|}{ Weight Percent of Size Fractions (in $\mathrm{mm})^{2}$} \\
\hline & & & & & $3-2$ & $2-1$ & $1-0.5$ & $0.5-0.25$ & $0.25-0.1$ & $0.1-0.05$ & $0.05-0.01$ & $0.01-0.005$ & $0.005-0.001$ & $<0.001$ \\
\hline $1-1,50-60$ & 22.40 & 15.3 & - & - & - & - & 1.43 & 6.43 & 24.33 & 22.37 & 23.88 & 6.74 & 5.45 & 9.37 \\
\hline $1-1,140-150$ & 12.30 & 41.8 & - & - & - & - & - & 3.01 & 5.45 & 5.53 & 9.43 & 6.67 & 21.54 & 48.37 \\
\hline $1-2,34-43$ & 13.36 & 91.8 & - & - & - & - & - & - & 0.90 & 1.42 & 0.52 & 23.29 & 28.29 & 45.58 \\
\hline $1-3,21-22$ & 4.28 & 18.5 & - & - & - & - & - & 0.26 & 62.85 & 1.94 & 5.61 & 7.38 & 8.64 & 13.32 \\
\hline $1-3,78-88$ & 15.14 & 89.5 & - & - & - & - & - & - & 0.13 & 1.06 & 0.73 & 29.99 & 25.62 & 42.47 \\
\hline $1-4,118-127$ & 14.52 & 60.1 & - & - & - & - & - & - & 0.14 & 0.28 & 0.28 & 23.82 & 37.74 & 37.74 \\
\hline $2-1,128-130$ & 11.95 & 43.8 & - & - & - & - & - & - & 3.10 & 2.76 & 4.52 & 29.20 & 30.21 & 30.21 \\
\hline $3-1,88-94$ & 11.99 & 34.1 & - & - & - & - & - & - & 0.75 & 2.00 & 6.26 & 26.26 & 23.61 & 41.12 \\
\hline $3-2,14-20$ & 23.45 & 20.2 & - & - & - & - & 2.86 & 11.68 & 20.51 & 14.12 & 33.01 & 4.13 & 5.63 & 8.06 \\
\hline $3-2,61-70$ & 7.37 & 51.5 & - & - & - & - & - & - & 0.27 & 1.36 & 3.12 & 13.97 & 25.65 & 55.63 \\
\hline $3, \mathrm{CC}$ & 5.04 & - & - & - & - & - & - & - & 4.96 & 2.18 & 0.79 & 9.53 & 26.19 & 56.35 \\
\hline $5-1,145-146$ & 5.98 & 23.8 & - & - & - & - & - & - & 0.33 & 0.33 & 0.16 & 36.96 & 31.61 & 30.61 \\
\hline $6-1,100-110$ & 9.34 & 38.8 & - & - & - & - & - & - & 1.82 & 1.07 & 1.82 & 18.41 & 38.44 & 38.44 \\
\hline $6-1,140-142$ & 11.79 & 36.9 & - & - & - & - & - & - & 0.17 & 0.17 & 0.51 & 21.88 & 24.09 & 53.18 \\
\hline $7-1,141-143$ & 8.05 & 37.8 & - & - & - & - & - & - & 1.24 & 0.12 & 1.24 & 21.25 & 30.56 & 45.59 \\
\hline $7-2,105-110$ & 6.69 & 39.9 & - & - & - & - & - & - & 1.05 & 3.74 & 30.02 & 5.24 & 9.87 & 50.08 \\
\hline $7-3,18-24$ & 14.75 & 34.6 & - & - & - & - & - & - & 1.63 & 3.66 & 2.03 & 33.23 & 35.86 & 23.59 \\
\hline $8-1,133-143$ & 12.60 & 28.9 & - & - & - & - & - & - & 0.16 & 0.16 & 0.79 & 16.67 & 41.11 & 41.11 \\
\hline $10-1,113-122$ & 20.31 & 22.0 & - & - & - & - & - & - & 0.34 & 0.89 & 3.25 & 48.06 & 30.72 & 16.74 \\
\hline $10-2,75-85$ & 21.74 & 26.0 & - & - & - & - & - & - & 0.64 & 4.55 & 0.60 & 28.70 & 32.52 & 32.99 \\
\hline $10-3,106-114$ & 2.56 & - & - & - & - & - & - & - & 0.39 & 0.39 & 0.39 & 31.01 & 33.91 & 33.91 \\
\hline $11-1,22-32$ & 1.85 & - & - & - & - & - & - & - & - & 0.54 & 1.62 & 31.78 & 32.81 & 33.25 \\
\hline $11-2,137-148$ & 15.20 & 29.7 & - & - & 3.95 & 1.05 & 0.26 & 0.20 & 1.18 & 1.05 & 7.50 & 20.99 & 24.87 & 38.95 \\
\hline $12-1,110-114$ & 17.87 & 28.5 & - & - & - & - & - & - & 0.11 & 0.45 & 0.56 & 12.84 & 31.72 & 54.32 \\
\hline $12-2,9-18$ & 10.67 & 43.1 & - & - & - & - & - & - & 0.09 & 1.69 & 2.91 & 16.03 & 39.64 & 39.64 \\
\hline $12-2,73-74$ & 2.05 & - & - & - & - & - & - & - & 0.49 & 2.44 & 6.34 & 25.47 & 32.63 & 32.63 \\
\hline $12-2,100-104$ & 18.34 & 22.4 & - & - & - & - & - & - & 0.05 & 0.05 & 0.49 & 22.53 & 25.79 & 51.09 \\
\hline $13-5,63-70$ & 7.59 & 32.2 & - & 5.40 & 14.08 & 12.52 & 0.92 & 0.26 & 0.26 & 0.40 & 0.92 & 8.06 & 28.59 & 28.59 \\
\hline $13-5,106-115$ & 1.98 & - & - & - & - & - & - & - & 0.51 & 0.51 & 3.54 & 31.10 & 33.43 & 30.91 \\
\hline $13-6,145-150$ & 6.81 & 48.8 & - & - & - & . - & - & - & 0.29 & 0.88 & 5.58 & 18.36 & 27.75 & 47.14 \\
\hline $14-2,1-8$ & 1.73 & - & - & - & - & - & - & - & - & 0.58 & 4.05 & 26.81 & 34.28 & 34.28 \\
\hline $14-2,65-73$ & 16.19 & 22.3 & - & - & - & - & - & - & 0.12 & 0.06 & 1.98 & 26.68 & 29.22 & 41.94 \\
\hline $14-2,128-135$ & 1.69 & - & - & - & - & - & - & - & 0.59 & 0.59 & 1.18 & 27.82 & 34.91 & 34.91 \\
\hline $15-1,52-60$ & 2.68 & - & - & - & - & - & - & - & 0.37 & 0.74 & 0.74 & 29.87 & 34.14 & 34.14 \\
\hline $15-2,91-100$ & 2.31 & - & - & - & - & - & - & - & 0.43 & 0.87 & 2.16 & 16.02 & 24.68 & 55.84 \\
\hline $15-3,29-36$ & 1.70 & - & - & - & - & - & - & - & - & 1.18 & 1.18 & 22.35 & 33.35 & 41.94 \\
\hline $15-4,54-63$ & 1.68 & - & - & - & - & - & - & - & - & 0.60 & 0.60 & 21.96 & 28.69 & 48.15 \\
\hline $15-5,89-98$ & 25.01 & 26.0 & - & - & - & - & - & - & 0.24 & 0.08 & 0.12 & 33.03 & 33.03 & 33.50 \\
\hline $15-6,19-26$ & 21.33 & 21.9 & - & - & - & - & - & - & 0.09 & 0.05 & 0.50 & 28.36 & 27.12 & 43.88 \\
\hline $16-1,57-62$ & 12.73 & 84.4 & - & - & - & - & - & - & 0.16 & 0.16 & 0.32 & 27.49 & 29.69 & 42.18 \\
\hline $16-3,32-37$ & 5.79 & 42.2 & - & - & - & - & - & - & 0.17 & 0.34 & 0.17 & 18.20 & 26.08 & 55.04 \\
\hline $16-4,83-92$ & 19.80 & 22.7 & - & - & - & - & - & - & 0.05 & 0.10 & 0.10 & 25.20 & 28.64 & 45.91 \\
\hline $18-2,80-86$ & 12.86 & 20.6 & - & - & - & - & - & - & 0.16 & 0.16 & 1.09 & 24.10 & 29.39 & 45.10 \\
\hline $18-3,130-139$ & 6.31 & 51.0 & - & - & - & - & - & - & 5.23 & 2.54 & 3.33 & 17.59 & 29.95 & 41.36 \\
\hline $18-4,115-124$ & 15.45 & 26.9 & - & - & - & - & - & - & 0.32 & 0.84 & 0.78 & 18.25 & 37.22 & 42.59 \\
\hline $18-5,14-20$ & 15.54 & 20.4 & - & - & - & - & - & - & 0.06 & 0.06 & 0.12 & 23.24 & 25.55 & 50.97 \\
\hline $18-5,65-73$ & 19.19 & 22.1 & - & - & - & - & - & - & 0.31 & 0.36 & 0.57 & 22.94 & 29.55 & 46.27 \\
\hline
\end{tabular}


TABLE 3

Grain Size of Sediments at Site 324 by the Combined Method

\begin{tabular}{|c|c|c|c|c|c|c|c|c|c|c|c|c|c|c|}
\hline \multirow{2}{*}{$\begin{array}{c}\text { Sample } \\
\text { (Interval in } \mathrm{cm} \text { ) }\end{array}$} & \multirow{2}{*}{$\begin{array}{c}\text { Weight } \\
\text { Suspension } \\
\text { (g) }\end{array}$} & \multirow{2}{*}{$\begin{array}{l}\% \text { of } \\
\text { Wet } \\
\text { Wt. }\end{array}$} & \multicolumn{12}{|c|}{ Weight Percent of Size Fractions (in mm) } \\
\hline & & & $>5$ & $5-3$ & $3-2$ & $2-1$ & $1-0.5$ & $0.5-0.25$ & $0.25-0.1$ & $0.1-0.05$ & $0.05-0.01$ & $0.01-0.005$ & $0.005-0.001$ & $<0.001$ \\
\hline $1-2,126-131$ & 6.29 & 52.8 & - & - & - & - & - & - & 2.07 & 1.59 & 2.23 & 18.11 & 30.05 & 45.95 \\
\hline $1-3,124-132$ & 8.86 & 45.8 & - & - & - & - & - & - & 2.48 & 0.79 & 4.85 & 16.15 & 21.33 & 54.40 \\
\hline $1-4,125-131$ & 10.16 & 36.4 & - & - & - & - & - & - & 0.10 & 0.20 & 2.76 & 20.86 & 24.21 & 51.87 \\
\hline $1-6,31-38$ & 13.91 & 38.3 & - & - & - & - & - & - & 2.59 & 0.93 & 1.22 & 19.69 & 24.46 & 51.11 \\
\hline $2-1,83-92$ & 13.91 & 34.0 & - & - & - & - & - & - & 0.07 & 0.07 & 0.80 & 21.64 & 27.17 & 50.25 \\
\hline $2-2,61-70$ & 15.97 & 66.9 & - & - & - & - & - & - & 0.06 & 0.06 & 1.13 & 14.03 & 35.50 & 49.22 \\
\hline $2-3,52-61$ & 9.19 & 34.7 & - & - & - & - & - & - & 1.85 & 0.22 & 1.96 & 14.47 & 26.77 & 54.73 \\
\hline $2-4,27-36$ & 10.70 & 33.5 & - & - & - & - & - & - & - & 0.09 & 3.36 & 14.74 & 26.39 & 55.42 \\
\hline $2-5,35-45$ & 9.41 & 33.8 & - & - & - & - & - & - & - & 0.21 & 2.55 & 19.66 & 30.18 & 47.40 \\
\hline $2-6,95-104$ & 9.98 & 39.7 & - & - & - & - & - & - & - & 0.10 & 1.60 & 16.13 & 22.55 & 59.62 \\
\hline $3-1,137-146$ & 12.35 & 25.3 & - & - & - & - & - & - & - & 0.08 & 0.64 & 19.28 & 30.61 & 49.39 \\
\hline $3-2,136-145$ & 12.03 & 32.7 & - & - & - & - & - & - & - & 0.08 & 0.72 & 23.47 & 23.61 & 52.12 \\
\hline $3-3,67-77$ & 14.20 & 28.7 & - & - & - & - & - & - & - & 0.07 & 0.21 & 25.56 & 26.62 & 47.54 \\
\hline $3-4,47-57$ & 12.91 & 37.5 & - & - & - & - & - & - & - & 0.08 & 1.32 & 27.03 & 29.28 & 42.29 \\
\hline $3-5,99-100$ & 12.80 & 31.5 & - & - & - & - & - & - & 0.16 & 0.16 & 2.11 & 22.19 & 22.80 & 52.58 \\
\hline $3-6,138-148$ & 13.79 & 28.1 & - & - & - & - & - & - & 0.36 & 1.16 & 6.02 & 31.47 & 27.41 & 33.58 \\
\hline $4-2,113-123$ & 14.63 & 30.3 & - & - & - & - & - & - & 0.07 & 0.07 & 2.26 & 23.44 & 24.54 & 49.62 \\
\hline $4-3,30-40$ & 10.63 & 30.0 & - & - & - & - & - & - & 0.09 & - & 1.41 & 16.00 & 31.98 & 50.52 \\
\hline $4-4,54-64$ & 13.42 & 27.3 & - & - & - & - & - & - & 0.07 & 0.07 & 2.91 & 19.52 & 25.34 & 52.09 \\
\hline $5-2,66-83$ & 14.03 & 35.7 & - & - & - & - & - & - & 0.29 & 0.15 & 15.32 & 13.47 & 20.24 & 50.53 \\
\hline $5-3,25-35$ & 16.64 & 81.3 & - & - & - & - & - & - & 0.06 & 0.12 & 3.13 & 20.13 & 34.07 & 42.49 \\
\hline $6-2,19-30$ & 17.00 & 27.2 & - & - & - & - & - & - & 0.18 & 0.76 & 4.82 & 19.30 & 33.35 & 41.59 \\
\hline $7-1,69-78$ & 15.85 & 26.0 & - & - & - & - & - & - & 0.06 & 0.06 & 1.39 & 19.31 & 29.84 & 49.34 \\
\hline $7-3,110-120$ & 16.41 & 14.6 & - & - & - & - & - & - & - & 0.07 & 2.44 & 6.63 & 34.55 & 56.31 \\
\hline $7-6,119-129$ & 12.99 & 25.0 & - & - & - & - & - & - & 0.08 & 0.08 & 3.00 & 8.85 & 29.10 & 58.89 \\
\hline $8-3,61-71$ & 13.67 & 27.0 & - & - & - & - & & - & 0.07 & 0.14 & 4.17 & 14.57 & 27.56 & 53.40 \\
\hline
\end{tabular}

TABLE 4

Grain Size of Sediments at Site 325 by the Combined Method

\begin{tabular}{|c|c|c|c|c|c|c|c|c|c|c|c|c|c|c|}
\hline \multirow{2}{*}{$\begin{array}{c}\text { Sample } \\
\text { (Interval in } \mathrm{cm} \text { ) }\end{array}$} & \multirow{2}{*}{$\begin{array}{l}\text { Weight } \\
\text { Suspension } \\
\text { (g) }\end{array}$} & \multirow{2}{*}{$\begin{array}{l}\% \text { of } \\
\text { Wet } \\
\text { Wt. }\end{array}$} & \multicolumn{12}{|c|}{ Weight Percent of Size Fractions (in mm) } \\
\hline & & & $>5$ & $5-3$ & $3-2$ & $2-1$ & $1-0.5$ & $0.5-0.25$ & $0.25-0.1$ & $0.1-0.05$ & $0.05-0.01$ & $0.01-0.005$ & $0.005-0.001$ & $<0.001$ \\
\hline $1-1,49-59$ & 13.33 & 34.8 & - & - & - & - & - & - & 5.03 & 0.60 & 2.93 & 19.87 & 28.36 & 43.21 \\
\hline $1-1,133-144$ & 9.36 & 43.6 & - & - & - & - & - & - & 0.64 & 0.32 & 2.67 & 31.30 & 34.29 & 30.78 \\
\hline $1-2,26-36$ & 15.57 & 41.8 & - & - & - & - & - & - & 0.76 & 0.28 & 2.18 & 29.52 & 35.76 & 31.50 \\
\hline $1-4,81-92$ & 17.91 & 25.6 & - & - & - & - & - & - & 5.58 & 5.64 & 13.23 & 25.19 & 31.66 & 18.70 \\
\hline $2-2,39-49$ & 16.78 & 24.6 & - & - & - & - & - & - & 5.07 & 2.98 & 3.58 & 25.79 & 31.29 & 31.29 \\
\hline $3-2,11-24$ & 13.82 & 27.3 & - & - & - & - & - & - & 0.36 & 0.58 & 3.62 & 26.84 & 34.30 & 34.30 \\
\hline $3-3,76-87$ & 19.56 & 22.6 & - & - & - & - & - & - & 3.17 & 4.45 & 19.17 & 22.81 & 25.20 & 25.20 \\
\hline $3-4,26-36$ & 16.34 & 22.5 & - & 1.71 & 1.65 & 2.14 & 2.51 & 3.06 & 2.88 & 5.75 & 16.40 & 7.90 & 17.38 & 38.62 \\
\hline $5-1,55-57$ & 15.34 & 31.2 & 5.60 & 0.78 & 0.78 & 0.72 & 0.06 & 0.18 & 0.52 & 0.60 & 1.17 & 23.95 & 24.64 & 40.00 \\
\hline $6-1,145-150$ & 11.25 & 29.0 & - & - & - & - & - & - & 16.33 & 0.36 & 3.64 & 14.85 & 33.60 & 46.22 \\
\hline $7-1,131-135$ & 13.25 & 14.8 & - & - & - & - & - & - & 1.96 & 3.92 & 8.08 & 8.45 & 18.57 & 59.02 \\
\hline $7-2,138-142$ & 12.32 & 18.6 & - & - & - & - & - & - & 0.65 & 0.97 & 3.57 & 15.51 & 30.68 & 48.62 \\
\hline $8-1,45-59$ & 12.35 & 21.5 & - & - & - & '- & - & - & 0.73 & 1.62 & 3.24 & 22.02 & 30.61 & 41.78 \\
\hline $8-2,119-128$ & 15.55 & 10.1 & - & - & - & - & - & - & 1.22 & 0.32 & 4.05 & 18.07 & 30.42 & 45.92 \\
\hline $8-3,27-35$ & 13.25 & 21.3 & - & - & - & - & - & - & 0.91 & 0.23 & 2.87 & 14.25 & 28.53 & 53.21 \\
\hline $9-3,90-98$ & 10.79 & 16.5 & - & - & - & - & - & - & 0.65 & 0.28 & 0.83 & 21.04 & 35.03 & 42.17 \\
\hline $10-1,35-39$ & 9.64 & 15.0 & - & - & - & - & - & - & 1.56 & 0.41 & 4.36 & 18.98 & 29.46 & 45.23 \\
\hline $10-2,26-30$ & 6.55 & 48.9 & - & - & 2.44 & 2.14 & 3.36 & 6.86 & 5.95 & 6.41 & 8.09 & 7.05 & 28.85 & 28.85 \\
\hline $10-3,99-102$ & 11.84 & 21.3 & - & - & - & - & - & - & 0.51 & 11.74 & 8.45 & 14.35 & 24.24 & 40.71 \\
\hline $10, \mathrm{CC}$ & 8.16 & 33.3 & - & - & - & - & - & - & 0.25 & 0.25 & 2.70 & 18.12 & 39.34 & 39.34 \\
\hline
\end{tabular}

ent, especially in the upper and middle parts of the unit. Nevertheless, the $M d$ values consistently range between 0.002 and $0.005 \mathrm{~mm}$. As noted by the steepness of the slope of the cumulative curves, the sediments are well sorted and, as in the overlying units, are primarily composed of clay-size particles.

Unit 4 (=Units 4 and 5 of the Site Report chapters, this volume) consists of 63 meters of (?) lower Oligocene through Upper Cretaceous sediments and is the lithologic extension of Unit 3. In Unit 4, however, the final aleuritic fraction is almost completely lacking. The entire section is represented by consolidated pelite, and in the lowermost part the finest pelitic fraction (less than $0.001 \mathrm{~mm}$ ) constitutes $45 \%-50 \%$ of the sediment.
All the Site 323 units (2, 3, and 4) are texturally and lithologically very similar and could be considered as a single lithologic unit.

\section{Site 324}

The shallowest hole drilled during DSDP Leg 35 was at Site 324, where 218 meters of Pliocene and Pleistocene deposits were recovered. The sediments are generally homogeneous, slightly consolidated and unconsolidated pelites, with a few interlayers of fine aleurite. In all samples, $70 \%-90 \%$ of the sediment is of pelitic size, and the fraction less than 0.001 constitutes from $45 \%$ to $50 \%$. The $M d$ values vary but little throughout the interval and do not exceed 0.001-0.002 mm. Inter- 
TABLE 5

Grain Size Analyses by Heavy Liquid $\left(2.9 \mathrm{~g} / \mathrm{cm}^{3}\right)$ Separation of the 0.05-0.1 mm Fraction at Site 322

\begin{tabular}{lccr}
\hline $\begin{array}{c}\text { Sample } \\
\text { Interval in cm) }\end{array}$ & $\begin{array}{c}\text { Weight } \\
\text { Suspension } \\
\text { (g) }\end{array}$ & $\begin{array}{c}\text { \% Heavy } \\
\text { Fraction }\end{array}$ & $\begin{array}{r}\text { \% Light } \\
\text { Fraction }\end{array}$ \\
\hline $1-2,26-30$ & 3.3176 & 1.20 & 98.80 \\
$1-3,70-76$ & 0.2018 & 1.88 & 98.12 \\
$2-2,113-116$ & 0.0311 & 5.46 & 94.54 \\
$3-1,18-20$ & 0.0132 & - & 100.00 \\
$4-1,65-67$ & 0.0848 & 1.17 & 98.83 \\
$4-2,57-60$ & 1.1013 & 0.30 & 99.70 \\
$5-1,81-85$ & 0.0711 & 0.98 & 99.02 \\
$6-1,30-33$ & 0.0255 & 5.09 & 94.91 \\
$9-2,143-146$ & 2.3503 & 0.15 & 99.85 \\
$10-1,101-105$ & 5.5209 & 1.21 & 98.79 \\
$10-2,43-46$ & 5.8622 & 2.36 & 97.64 \\
$11-1,42-46$ & 6.7072 & 3.61 & 96.39 \\
$11-2,45-49$ & 5.1030 & 3.80 & 96.20 \\
$11-3,24-32$ & 4.5875 & 4.94 & 95.06 \\
$11-4,17-24$ & 4.0727 & 5.20 & 94.80 \\
$11-5,36-40$ & 0.0330 & 9.39 & 90.61 \\
$11-6,109-115$ & 0.0274 & 2.55 & 97.45 \\
$13-2$ & 0.0337 & 2.96 & 97.04 \\
\hline
\end{tabular}

TABLE 6

Grain Size Analyses by Heavy Liquid $\left(2.9 \mathrm{~g} / \mathrm{cm}^{3}\right)$ Separation of the 0.05-0.1 mm Fraction at Site 323

\begin{tabular}{|c|c|c|c|}
\hline $\begin{array}{c}\text { Sample } \\
\text { (Interval in cm) }\end{array}$ & $\begin{array}{l}\text { Weight } \\
\text { Suspension } \\
\text { (g) }\end{array}$ & $\begin{array}{l}\text { \% Heavy } \\
\text { Fraction }\end{array}$ & $\begin{array}{l}\% \text { Light } \\
\text { Fraction }\end{array}$ \\
\hline $1-1,50-60$ & 5.0165 & 1.84 & 98.16 \\
\hline $1-1,140-150$ & 0.6837 & 1.59 & 98.41 \\
\hline $1-2,34-43$ & 0.1974 & 0.45 & 99.55 \\
\hline $1-3,21-22$ & 0.8373 & 7.01 & 92.99 \\
\hline $1-3,78-88$ & 0.1617 & - & 100.00 \\
\hline $1-4,118-127$ & 0.0389 & 0.25 & 99.75 \\
\hline $2-1,128-130$ & 0.3330 & - & 100.00 \\
\hline $3-1,88-94$ & 0.2456 & - & 100.00 \\
\hline $3-2,14-20$ & 3.3150 & 2.84 & 97.16 \\
\hline $3-2,61-70$ & 0.0955 & - & 100.00 \\
\hline $3-\mathrm{CC}$ & 0.1171 & 2.04 & 97.96 \\
\hline $6-1,100-110$ & 0.1032 & - & 100.00 \\
\hline $7-2,105-110$ & 0.2566 & - & 100.00 \\
\hline $7-3,18-22$ & 0.5566 & - & 100.00 \\
\hline $10-1,119-112$ & 0.1836 & - & 100.00 \\
\hline $10-2,26-30$ & 0.4228 & 7.23 & 92.77 \\
\hline $10-2,75-85$ & 1.0069 & - & 100.00 \\
\hline $10-3,99-102$ & 1.3914 & 6.34 & 93.66 \\
\hline $10-3,106-114$ & 0.2875 & - & 100.00 \\
\hline $11-1,22-32$ & 0.2409 & - & 100.00 \\
\hline $11-2,137-148$ & 0.1678 & 0.65 & 99.35 \\
\hline $12-1,110-114$ & 0.0885 & - & 100.00 \\
\hline $12-2,9-18$ & 0.6611 & 0.49 & 99.51 \\
\hline $12-2,73-77$ & 0.1526 & - & 100.00 \\
\hline $13-5,106-115$ & 0.2745 & - & 100.00 \\
\hline $13-6,145-150$ & 0.1677 & 0.44 & 99.56 \\
\hline $14-2,1-8$ & 0.1677 & - & 100.00 \\
\hline $15-1,52-60$ & 0.0435 & 1.60 & 98.40 \\
\hline $15-2,91-100$ & 0.0234 & - & 100.00 \\
\hline $15-3,25-36$ & 0.0123 & - & 100.00 \\
\hline $15-4,54-69$ & 0.0121 & - & 100.00 \\
\hline $15-5,89-98$ & 0.0210 & 2.85 & 97.15 \\
\hline $15-6,19-26$ & 0.0111 & - & 100.00 \\
\hline $16-1,57-62$ & 0.0335 & 0.29 & 99.71 \\
\hline $16-3,32-37$ & 0.0011 & - & 100.00 \\
\hline $16-4,83-92$ & 0.0222 & 17.56 & 82.44 \\
\hline $18-2,80-86$ & 0.0081 & - & 100.00 \\
\hline $18-3,130-139$ & 0.1681 & - & 100.00 \\
\hline $18-4,115-124$ & 0.1352 & 1.18 & 98.82 \\
\hline $18-5,65-73$ & 0.0698 & 8.59 & 91.41 \\
\hline
\end{tabular}

TABLE 7

Grain Size Analyses by Heavy Liquid $\left(2.9 \mathrm{~g} / \mathrm{cm}^{3}\right)$ Separation of the $0.05-0.1 \mathrm{~mm}$ Fraction at Site 324

\begin{tabular}{lccc}
\hline $\begin{array}{c}\text { Sample } \\
\text { (Interval in cm) }\end{array}$ & $\begin{array}{c}\text { Weight } \\
\text { Suspension } \\
\text { (g) }\end{array}$ & $\begin{array}{c}\text { \% Heavy } \\
\text { Fraction }\end{array}$ & $\begin{array}{c}\text { \% Light } \\
\text { Fraction }\end{array}$ \\
\hline $1-2,126-131$ & 0.1083 & 1.66 & 98.34 \\
$1-6,31-38$ & 0.1468 & 2.99 & 97.01 \\
\hline
\end{tabular}

TABLE 8

Grain Size Analyses by Heavy Liquid $\left(2.9 \mathrm{~g} / \mathrm{cm}^{3}\right)$ Separation of the $0.05-0.1 \mathrm{~mm}$ Fraction at Site 325

\begin{tabular}{lcrr}
\hline $\begin{array}{c}\text { Sample } \\
\text { (Interval in cm) }\end{array}$ & $\begin{array}{c}\text { Weight } \\
\text { Suspension } \\
(\mathrm{g})\end{array}$ & $\begin{array}{c}\text { \% Heavy } \\
\text { Fraction }\end{array}$ & $\begin{array}{r}\text { \% Light } \\
\text { Fraction }\end{array}$ \\
\hline $1-1,49-59$ & 0.0900 & 3.55 & 96.45 \\
$1-1,133-144$ & 0.0269 & - & 100.00 \\
$1-2,26-36$ & 0.374 & 4.27 & 95.73 \\
$1-4,81-92$ & 1.0097 & 1.72 & 98.28 \\
$2-2,39-49$ & 0.4026 & 5.06 & 94.94 \\
$3-2,11-24$ & 0.0855 & 2.10 & 97.90 \\
$3-3,76-87$ & 0.8808 & 3.06 & 96.94 \\
$3-4,26-36$ & 0.9432 & 4.62 & 95.38 \\
$5-1,55-57$ & 0.0099 & 57.57 & 42.43 \\
5, CC & 0.0138 & 5.79 & 94.21 \\
$6-1,145-150$ & 0.0506 & 4.54 & 95.46 \\
$7-1,131-135$ & 0.5280 & 4.96 & 95.04 \\
$7-2,138-142$ & 0.1262 & 1.66 & 98.34 \\
$8-1,45-49$ & 0.0370 & 3.51 & 96.49 \\
$8-2,119-128$ & 0.0560 & 1.78 & 98.22 \\
$8-3,27-35$ & 0.0292 & - & 100.00 \\
$9-3,90-98$ & 0.0281 & 2.49 & 97.51 \\
$10-1,35-39$ & 0.0437 & 1.60 & 98.40 \\
\hline
\end{tabular}

TABLE 9

Grain Size of Sediments of Site 325, Core 8, Section 2 , by Heavy Liquid $\left(2.9 \mathrm{~g} / \mathrm{cm}^{3}\right)$ Separation of the 0.05-0.1 mm Fraction

\begin{tabular}{ccrc}
\hline $\begin{array}{c}\text { Sample } \\
\text { Interval } \\
(\mathrm{cm})\end{array}$ & $\begin{array}{c}\text { Weight } \\
\text { Suspension } \\
(\mathrm{g})\end{array}$ & $\begin{array}{c}\text { \% Heavy } \\
\text { Fraction }\end{array}$ & $\begin{array}{c}\text { \% Light } \\
\text { Fraction }\end{array}$ \\
\hline $22.5-23.5$ & 2.6661 & 3.23 & 96.77 \\
$23.5-25.0$ & 3.6730 & 3.74 & 96.26 \\
$25.0-26.0$ & 2.1825 & 5.27 & 94.73 \\
$26.0-27.5$ & 3.9642 & 10.23 & 89.77 \\
$27.5-29.0$ & 2.6561 & 4.86 & 95.14 \\
$29.0-30.5$ & 2.3684 & 6.08 & 93.92 \\
$30.5-32.0$ & 2.1134 & 7.68 & 92.32 \\
$32.0-33.5$ & 1.5235 & 9.98 & 90.02 \\
$33.5-34.5$ & 1.2390 & 9.56 & 90.44 \\
$34.5-35.5$ & 1.4693 & 6.62 & 93.38 \\
$35.5-37.0$ & 0.8729 & 10.21 & 89.79 \\
$37.0-39.0$ & 1.5448 & 8.52 & 91.48 \\
$39.0-41.0$ & 1.4208 & 8.82 & 91.18 \\
$41.0-42.0$ & 0.1744 & 5.96 & 94.04 \\
$42.0-44.0$ & 0.0373 & 2.14 & 97.86 \\
\hline
\end{tabular}

bedded consolidated pelitic sediments occur in the lower part of the section and are underlain by a zone of loose sand. This pattern somewhat resembles that of the uppermost part of Unit 2, of Site 323. However, because these intervals are of different ages, a parallel cannot be drawn. The sediments penetrated at Site 324 correlate with those of the uppermost units of Sites 322 and 323. 
TABLE 10

Grain Size of Elutriated Samples of Sites 322-325 Separated by Heavy Liquid $\left(2.9 \mathrm{~g} / \mathrm{cm}^{3}\right)$ of the 0.1-0.05 mm Fraction

\begin{tabular}{lcccr}
\hline $\begin{array}{c}\text { Sample } \\
\text { (Interval in cm) }\end{array}$ & $\begin{array}{c}\text { Sample } \\
\text { Weight } \\
(\mathrm{g})\end{array}$ & $\begin{array}{c}\text { Weight } \\
\text { Suspension } \\
(\mathrm{g})\end{array}$ & $\begin{array}{c}\text { \% Heavy } \\
\text { Fraction }\end{array}$ & $\begin{array}{r}\text { \% Light } \\
\text { Fraction }\end{array}$ \\
\hline Site 322 & & & & \\
1, CC & 0.8334 & 0.0182 & 7.14 & 92.86 \\
2, CC & 0.2770 & 0.1356 & 1.40 & 98.60 \\
3, CC & 0.0236 & 0.0101 & - & 100.00 \\
4, CC & 0.0918 & 0.0686 & 1.60 & 98.40 \\
5, CC & 0.1695 & 0.0987 & 1.62 & 98.38 \\
6, CC & 0.3299 & 0.1811 & 5.85 & 94.15 \\
10, CC & 0.5084 & 0.3208 & 1.77 & 98.23 \\
11, CC & 0.3451 & 0.2303 & 10.37 & 89.63
\end{tabular}

Site 323

$\begin{array}{lrrrr}\text { 1, CC } & 0.0763 & 0.0345 & - & 100.00 \\ \text { 2, CC }(>63 \mu \mathrm{m}) & 1.3282 & 0.1370 & 2.34 & 97.66 \\ \text { 3, CC } & 0.2479 & 0.1092 & 2.01 & 97.99 \\ \text { 4, CC }(>63 \mu \mathrm{m}) & 0.1562 & 0.0129 & 6.20 & 93.80 \\ \text { 5, CC } & 0.0931 & 0.0472 & 1.69 & 98.31 \\ \text { 6, CC } & 0.0508 & 0.0208 & 0.48 & 99.52 \\ \text { 7, CC } & 2.2319 & 1.1507 & 1.74 & 98.26 \\ \text { 8, CC } & 0.3887 & 0.0840 & 4.05 & 95.95 \\ \text { 9, CC } & 0.0175 & 0.0028 & - & 100.00 \\ \text { 10, CC } & 0.0712 & 0.0175 & - & 100.00 \\ \text { 12, CC } & 0.0230 & 0.0156 & 68.58 & 31.42 \\ \text { 13, CC } & 0.0294 & 0.0213 & 67.13 & 32.87 \\ \text { 15, CC } & 0.0342 & 0.0222 & - & 100.00 \\ \text { 17, CC } & 0.0210 & 0.0110 & 70.90 & 29.10 \\ \text { 8-3 } & 0.3603 & 0.1661 & 0.06 & 99.94\end{array}$

Site 324

$\begin{array}{lllll}1-2 & 1.0611 & 0.8310 & 2.64 & 97.36 \\ 1-3 & 0.2339 & 0.1483 & 1.15 & 98.85 \\ 3, \text { CC } & 0.1412 & 0.0187 & 3.74 & 96.26 \\ 4, \text { CC } & 0.0072 & 0.0037 & 2.70 & 97.30 \\ 5, \text { CC } & 0.0621 & 0.0560 & 0.18 & 99.82 \\ 6, \text { CC } & 0.0076 & 0.0051 & 1.96 & 98.04 \\ 7, \text { CC } & 0.1035 & 0.0585 & 4.44 & 95.56 \\ 8-2 & 0.5268 & 0.1617 & 2.41 & 97.59\end{array}$

Site 325

\begin{tabular}{lrrrr}
$1-4$ & 12.3110 & 0.8792 & 10.99 & 89.01 \\
$2-1$ & 1.2225 & 0.3388 & 3.01 & 96.99 \\
$2, \mathrm{CC}$ & 0.1374 & 0.0810 & 0.86 & 99.14 \\
$3-1$ & 0.9659 & 0.0156 & 13.46 & 86.54 \\
$4, \mathrm{CC}$ & 0.2334 & 0.0744 & 1.48 & 98.52 \\
$3, \mathrm{CC}$ & 0.2956 & 0.0576 & 1.39 & 98.61 \\
$4-1$ & 1.1658 & 0.0100 & 5.00 & 95.00 \\
$4-1$ & 0.7744 & 0.1066 & 2.06 & 97.94 \\
$7-2$ & 0.1362 & 0.0654 & 0.15 & 99.85 \\
$9-3$ & 0.0862 & 0.0325 & 0.31 & 99.69 \\
$9, \mathrm{CC}$ & 0.2775 & 0.0902 & 0.11 & 99.89 \\
$10-3$ & 6.1549 & 3.7293 & 4.89 & 95.11 \\
$10-3$ & 1.2168 & 0.4261 & 3.49 & 96.51 \\
\hline
\end{tabular}

\section{Site 325}

In comparison with the other sites, the sampling of sediments at Site 325 was considerably poorer; as a consequence, we succeeded in tentatively defining only two units with diverse lithological features.

Unit 1 consists of a thick sequence (approximately $600-620 \mathrm{~m}$ ) of intercalated soft and more consolidated pelites, with an admixture of fine aleurite in the upper part and consolidated pelites with a small admixture of fine aleurite in the lowermost part of the section. The sediments have tentatively been dated as ? Oligocene to Pliocene. In the upper, soft part, sorting is poor to moderate (So fluctuates from 2 to 3.16). The $M d$ values, which vary from 0.002 to $0.005 \mathrm{~mm}$, indicate the presence of a great amount of fine-grained material. The content of pelitic particles averages $80 \%-90 \%$ of which the finest fraction comprises $15 \%-60 \%$. Ice-rafted debris is common in the sediments of this unit.

Unit 2 is 100 meters thick and is characterized by a greater degree of consolidation. It consists of intercalated sandstones, aleurites, and pelites of lower Miocene to (?) Oligocene age and does not differ greatly in grain size from Unit 1 . It is noteworthy, however, that the percentage of the fraction less than $0.001 \mathrm{~mm}$ is consistent throughout the unit and almost always forms $40 \%-50 \%$ of the sample. The $M d$ values do not differ from those of Unit 1 and are 0.001-0.004 mm. Most of the sediments are well sorted.

\section{MINERALOGY OF COARSE ALEURITIC FRACTIONS}

We studied the mineralogy of the aleuritic fraction (grain size larger than $0.05 \mathrm{~mm}$ ) in great detail. This size fraction is widely distributed in bottom sediments, and its composition provides the best basis for recognition of mineral suites and the presence of heavy minerals.

Two methods were employed in preparation of the samples for analyses. We prepared washed residues of the most representative sediments (over $50 \mathrm{~g}$ ) by means of a $0.063 \mathrm{~mm}$ sieve onboard ship in order to immediately obtain mineralogical data. In addition, the fraction larger than $0.05 \mathrm{~mm}$ was isolated during grain-size analysis. We isolated the heavy subfraction by separation with heavy liquid with a specific gravity of 2.90 . The ratios of the heavy to light subfractions are shown in Tables 5-10. The percentage of the heavy subfraction is not constant, but varies between $1 \%$ and $71 \%$; in most cases, however, it is quite low.

The distribution of minerals found in the light and heavy fractions is presented in Tables 11-14. These data show that various sedimentary, metamorphic, and igneous rocks were the sources for the clastic minerals. Biogenic and authigenic minerals were also very significant in the origin of the sediments.

\section{Site 322}

The most common minerals of the heavy subfraction of the Neogene sediments are monoclinic pyroxene, epidote, and opaque minerals. Rock debris, altered minerals, hornblende, and orthopyroxenes (mainly hypersthenes) are less common. The abundance of these clastic minerals is considerably lower in the sediments near the base of the hole; here the authigenic hydrous ferric oxides and manganese are the major constituents.

The content of pyrite, another authigenic mineral, varies from single grains to $2.9 \%$ and markedly increases in the overlying Miocene sediments. Accessory minerals form the remainder of the heavy subfraction.

\section{Site 323}

The mineral suites of the Neogene sediments from the upper part of this hole differ from those found at 
TABLE 11A

Distribution of Minerals in the Heavy and Light Fraction of Leg 35 Sediments

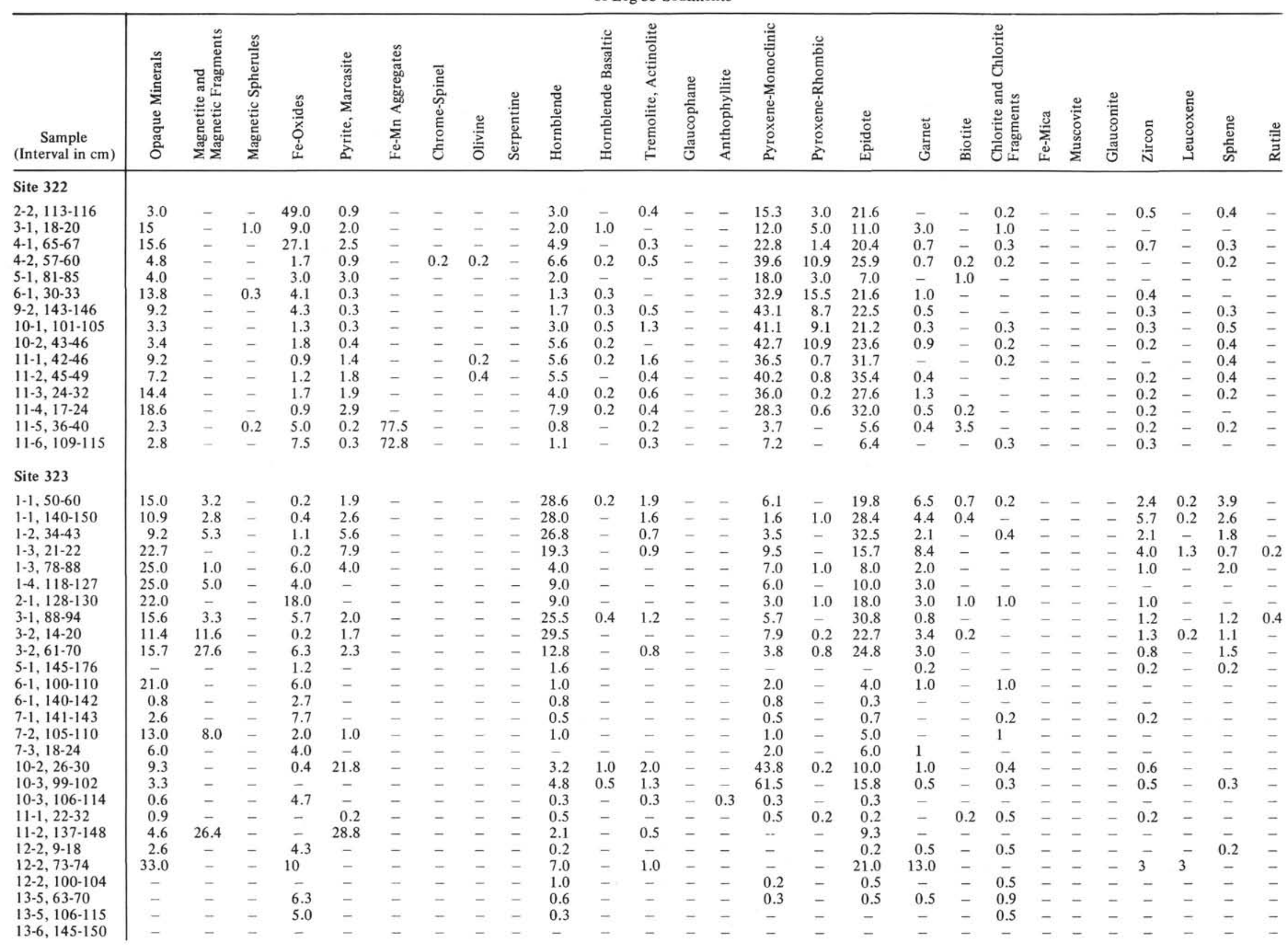




\begin{tabular}{|c|c|c|c|c|c|c|c|c|c|c|c|c|c|c|c|c|c|c|c|c|c|c|c|c|c|c|c|}
\hline $14-2,1-8$ & - & - & - & 5.4 & - & - & - & - & - & 0.6 & - & - & - & - & 0.3 & - & 0.6 & - & - & 1.3 & - & - & - & - & - & - & $\overline{-}$ \\
\hline $14-2,65-73$ & - & - & - & 3.5 & - & - & - & - & - & 0.5 & - & - & - & - & - & - & 1.4 & - & - & 0.5 & - & - & - & - & - & - & - \\
\hline $14-2,128-135$ & - & - & - & 12.9 & - & - & - & - & - & - & - & - & - & - & - & - & - & - & - & 0.3 & - & - & - & - & - & - & - \\
\hline $15-1,52-60$ & - & - & - & 12.3 & - & - & - & - & - & - & - & - & - & - & - & - & - & - & - & - & - & - & - & - & - & - & - \\
\hline $15-2,91-100$ & - & - & - & 4.5 & - & - & - & - & - & - & - & - & - & - & - & - & - & - & - & - & - & - & - & - & - & - & - \\
\hline $15-3,29-36$ & 0.7 & - & - & 9.1 & - & - & - & - & - & - & - & - & - & - & 0.4 & - & 0.2 & - & - & - & - & - & - & 0.4 & - & - & - \\
\hline $15-5,89-92$ & - & - & - & - & - & - & - & - & - & - & - & - & - & - & - & - & - & - & - & - & - & - & - & - & - & - & - \\
\hline $14-6,19-26$ & 5.0 & - & - & 1.0 & - & - & - & - & - & - & - & - & - & - & 3.0 & - & 2.0 & 1.0 & - & - & - & - & - & - & - & - & - \\
\hline $16-1,57-62$ & $90.0 ?$ & - & - & 2.9 & - & - & - & - & - & 0.8 & - & - & - & - & 1.3 & - & 3.3 & - & - & - & - & - & - & - & - & - & - \\
\hline $16-3,32-37$ & 7.4 & - & - & 21.5 & - & 67.4 & - & - & - & - & - & - & - & - & - & - & - & - & - & - & - & - & - & - & - & - & - \\
\hline $16-4,88-93$ & 10.2 & 1.3 & - & 2.6 & - & 8.1 & - & - & - & - & - & - & - & - & 0.3 & 0.3 & 0.5 & 0.5 & - & - & - & - & - & 0.5 & 0.3 & - & - \\
\hline $18-2,80-86$ & 6.0 & - & - & 8.0 & - & - & -. & - & - & 1.0 & - & - & - & - & - & - & 1.0 & - & - & - & - & - & - & 1 & - & - & - \\
\hline $18-3,130-139$ & 5 & - & - & 5 & - & - & - & - & - & - & - & - & - & - & 2 & - & 3 & - & - & - & - & - & - & 2 & - & -. & - \\
\hline $18-4,115-124$ & 1.7 & - & - & 0.9 & 0.4 & 93.3 & - & - & - & - & - & - & - & - & - & - & - & - & - & - & - & - & - & 0.4 & - & - & - \\
\hline $18-5,14-20$ & - & - & - & 3.2 & - & - & - & - & - & - & - & - & - & - & 0.7 & 0.2 & 0.9 & - & - & 0.7 & - & - & - & - & - & - & - \\
\hline $18-5,65-73$ & - & - & - & 10.7 & - & 87.7 & - & - & - & - & - & - & - & - & - & - & - & 0.3 & - & - & - & - & - & 0.3 & - & - & - \\
\hline $18-5,133-143$ & 0.6 & - & - & 1.5 & - & - & - & - & - & - & - & - & - & - & - & - & 0.3 & - & - & - & - & - & - & - & - & - & - \\
\hline $3, \mathrm{CC}$ & 12.8 & $=$ & - & 3.1 & 3.8 & - & - & - & - & 30.9 & - & - & - & - & 6.5 & 0.4 & 26.2 & 4.2 & - & - & - & - & - & 4.9 & - & 0.8 & - \\
\hline \multicolumn{28}{|l|}{ Site 324} \\
\hline $1-2,126-131$ & 15 & - & - & 4 & - & - & - & - & - & 19 & - & - & - & - & 23 & 1. & 6 & 3 & - & 2 & - & - & - & 1 & 2 & 1 & - \\
\hline $1-4,125-131$ & 1.0 & - & - & 4.0 & - & - & - & - & - & 1.0 & - & - & - & - & 0.3 & - & 0.5 & 0.5 & - & - & - & - & - & 0.3 & - & - & - \\
\hline $1-6,31-38$ & 31.2 & - & - & 0.5 & - & - & - & - & - & 33.8 & - & - & - & - & 3.9 & 1.3 & 9.7 & 4.7 & - & - & - & - & - & 1.8 & 0.5 & 1.6 & 0.5 \\
\hline $2-1,83-92$ & 0.3 & - & - & 0.3 & - & - & - & - & - & 1.8 & - & 0.3 & - & - & 1.2 & 0.3 & 0.8 & - & - & 1.2 & - & - & - & - & - & - & - \\
\hline $2-2,61-70$ & 0.3 & - & - & 0.3 & - & - & - & - & - & - & - & - & - & - & - & - & 0.3 & 0.3 & - & - & - & - & - & - & - & - & - \\
\hline $2-3,52-61$ & 1.4 & - & - & 0.3 & 1.1 & - & - & - & - & 1.1 & - & - & - & - & 0.8 & - & 1.4 & - & - & 3.1 & - & - & - & 0.6 & - & - & - \\
\hline $2-4,27-36$ & 0.9 & - & - & 5.0 & - & - & - & - & - & 0.2 & - & - & - & - & - & - & 0.4 & - & 0.7 & - & - & - & - & 0.2 & - & - & - \\
\hline $2-5.35-45$ & 3.6 & - & - & 1.0 & - & - & - & - & - & 0.3 & - & - & - & - & 0.3 & - & - & - & - & 3.6 & - & - & - & - & - & - & - \\
\hline $2-6.95-104$ & 1.7 & - & - & 0.6 & - & - & - & - & - & - & - & - & - & - & - & - & - & - & - & - & - & - & - & - & - & - & - \\
\hline $3-1,137-146$ & 25.5 & - & - & 3.2 & - & - & - & - & - & 0.7 & - & - & - & - & - & - & 1.1 & - & - & - & - & - & - & - & - & - & - \\
\hline $3-2,136-145$ & 2.8 & - & - & 6.3 & - & - & - & - & - & 0.5 & - & - & - & - & 1.0 & 1.0 & 0.5 & 1.0 & - & - & - & - & - & 0.5 & - & - & - \\
\hline $3-3,67-77$ & 5.6 & - & - & 4.7 & - & - & - & - & - & 0.6 & - & - & - & - & 0.3 & - & 0.8 & - & - & - & - & - & - & 0.5 & - & - & - \\
\hline $3-4.47-57$ & 1.1 & - & - & 2.8 & - & - & - & - & - & 0.4 & - & - & - & - & - & - & 0.4 & 0.4 & - & - & - & - & - & 0.4 & - & - & - \\
\hline $3-5.99-100$ & - & - & - & 2.5 & - & - & - & - & - & 0.5 & - & - & - & - & 0.5 & - & 0.7 & 0.2 & - & 0.2 & - & - & - & - & - & - & - \\
\hline $4-2.113-123$ & 2.9 & - & - & 4.2 & - & - & - & - & - & 3.2 & - & - & - & - & - & - & - & - & - & 1.6 & - & - & - & - & - & - & - \\
\hline $4-3.30-40$ & - & - & - & - & - & - & - & - & - & 1.8 & - & - & - & - & 0.9 & - & 0.5 & - & 0.5 & 1.3 & - & - & - & - & - & - & - \\
\hline $4-4,54-64$ & 0.8 & - & - & 3.6 & - & - & - & - & - & 0.4 & - & - & - & - & 1.1 & - & 0.4 & 0.4 & - & - & - & - & - & - & - & - & - \\
\hline $5-2,66-83$ & 0.6 & - & - & 5.4 & - & - & - & - & - & 0.6 & - & - & - & - & - & - & - & - & 0.4 & 0.2 & - & - & - & - & - & - & - \\
\hline $5-3.25-35$ & - & - & - & - & - & - & - & - & - & - & - & - & - & - & - & - & - & - & - & - & - & - & - & - & - & - & - \\
\hline $7-1,69-7$ & - & - & - & - & - & - & - & - & - & 1 & - & - & - & - & 1 & - & - & - & 1 & - & - & - & - & 1 & - & - & - \\
\hline $7-3.110-120$ & 7.0 & - & - & 8.6 & - & - & - & - & - & 0.5 & - & - & - & - & - & 0.3 & 0.5 & 0.3 & - & 0.3 & - & - & - & - & - & 0.3 & - \\
\hline $7-6,119-129$ & 0.9 & - & - & 22.5 & 1.1 & - & - & - & - & 0.3 & - & - & - & - & 0.3 & - & 0.3 & - & - & 0.3 & - & - & - & - & - & - & - \\
\hline $8-3.61-71$ & 13.6 & - & - & 0.3 & - & - & - & - & - & 3.0 & - & - & - & - & 0.3 & 0.3 & 0.5 & - & - & 3.8 & - & - & - & - & - & - & - \\
\hline \multicolumn{28}{|l|}{ Site 325} \\
\hline $1-1.49-59$ & 12.3 & 9.4 & - & 3.4 & 0.5 & - & - & - & - & 22.1 & - & 0.5 & - & - & 7.8 & 4.7 & 28.6 & 0.9 & - & 0.5 & - & - & - & 0.5 & 0.3 & 0.3 & - \\
\hline $1-1,133-144$ & 8.3 & 13.0 & - & 3.2 & - & 13.5 & - & - & - & 16.2 & - & 1.4 & - & - & 11.1 & 3.2 & 18.4 & 2.3 & - & - & - & - & - & 0.5 & - & - & - \\
\hline $1-2,26-36$ & 43 & - & - & - & - & - & - & - & - & 4 & - & - & - & - & 5 & 2 & 14 & 1 & - & - & - & - & - & - & - & - & - \\
\hline $1-4,181-192$ & 15.9 & - & - & 3.4 & 0.2 & - & - & - & - & 27.7 & 0.2 & - & - & - & 20.8 & 1.8 & 21.9 & 0.2 & - & 0.2 & - & - & - & - & - & 0.7 & - \\
\hline $2-2,39-49$ & 18.2 & - & - & 0.4 & 0.2 & - & - & - & - & 22.9 & 0.2 & 2.5 & - & - & 19.3 & 0.8 & 24.5 & 0.4 & - & 0.6 & - & - & - & 0.6 & - & 0.4 & - \\
\hline $3-2,11-24$ & 19.7 & - & - & 3.3 & 2.9 & - & - & - & - & 22.2 & - & - & - & - & 15.3 & 3.6 & 23.4 & 0.4 & - & 0.4 & - & - & - & 1.4 & - & - & 0.4 \\
\hline $3-3,76-87$ & 11.5 & - & - & 0.2 & 0.2 & - & - & - & - & 27.1 & - & 0.9 & - & - & 26.0 & 0.9 & 19.9 & - & - & 0.2 & - & - & - & 0.7 & - & 0.2 & - \\
\hline $3-4,26-36$ & 15.4 & - & - & 0.2 & 3.4 & - & - & - & - & 27.8 & - & 1.9 & - & - & 16.8 & 1.5 & 24.9 & 0.2 & - & - & - & - & - & 0.7 & 0.5 & 0.2 & - \\
\hline $5-1,55-57$ & 18.8 & - & - & 1.6 & 1.0 & - & - & - & - & 6.5 & 0.8 & 0.3 & - & - & 32.9 & 4.9 & 23.8 & 2.1 & - & - & - & - & - & 0.8 & 1.3 & 0.3 & - \\
\hline $5, \mathrm{CC}$ & 43.4 & - & - & 1.0 & - & - & - & - & - & - & - & - & - & - & 38.3 & - & 7.7 & 1.9 & - & - & - & - & - & 1.9 & - & - & - \\
\hline
\end{tabular}


TABLE 11A - Continued

\begin{tabular}{|c|c|c|c|c|c|c|c|c|c|c|c|c|c|c|c|c|c|c|c|c|c|c|c|c|c|c|c|}
\hline $\begin{array}{c}\text { Sample } \\
\text { (Interval in } \mathrm{cm} \text { ) }\end{array}$ & 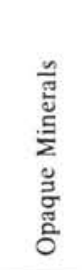 & 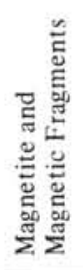 & 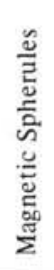 & 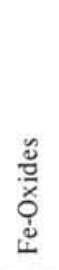 & 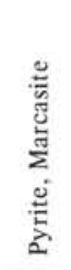 & 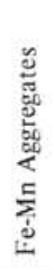 & $\begin{array}{l}\bar{\Xi} \\
\text { की } \\
\text { के } \\
\text { हू } \\
\text { है } \\
\text { है }\end{array}$ & 竞 & 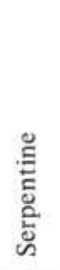 & 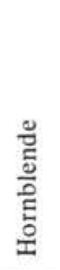 & 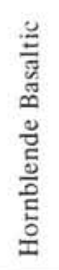 & 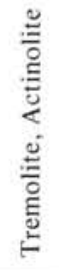 & 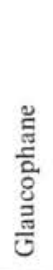 & 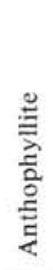 & 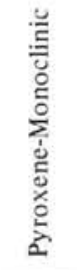 & 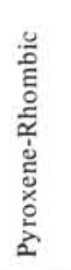 & : & $\begin{array}{l}\vec{\Xi} \\
\text { E్ }\end{array}$ & 芯 & 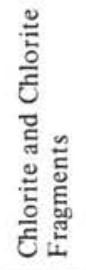 & 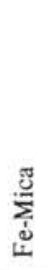 & 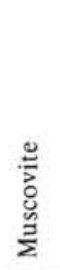 & 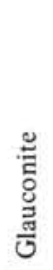 & 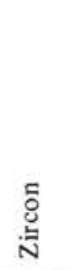 & 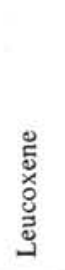 & $\begin{array}{l}\text { एँ } \\
\text { ஸे }\end{array}$ & $\stackrel{\Xi}{\rightleftarrows}$ \\
\hline $6-1,145-150$ & 25.3 & - & - & 0.9 & 21.0 & - & - & - & - & 1.4 & 0.2 & 0.9 & - & - & 36.9 & 1.4 & 6.4 & 0.7 & - & - & - & - & - & 0.2 & 0.2 & 0.2 & \\
\hline $7-1,131-134$ & 14.5 & - & - & 1.8 & - & - & - & - & - & 6.8 & 0.2 & - & - & - & 53.2 & 0.2 & 13.5 & 2.3 & - & 0.2 & - & - & - & 0.5 & 0.7 & - & - \\
\hline $7-2,138-142$ & 20.0 & - & - & 0.8 & 0.8 & - & - & - & - & 1.0 & - & 0.5 & - & - & 48.0 & 0.3 & 18.0 & 2.5 & - & - & - & - & - & 0.3 & - & 0.3 & - \\
\hline $8-1,45-49$ & 33.5 & - & - & 3.9 & 8.7 & - & - & - & - & 6.8 & 1.0 & - & - & - & 15.4 & - & 9.5 & 12.5 & - & - & - & - & - & 1.9 & - & 1.0 & - \\
\hline $8-2,22,5-23,5$ & 8.9 & - & - & 0.3 & - & - & - & - & - & 1.7 & 1.1 & - & - & - & 47.8 & 0.6 & 19.7 & 0.8 & - & - & - & _- & - & 0.6 & - & - & - \\
\hline $8-2,23,5-25,0$ & 12.9 & - & - & 1.4 & 3.5 & - & - & - & - & 1.8 & 2.5 & - & - & - & 46.0 & 0.4 & 14.6 & 0.7 & - & 1.6 & - & - & - & 0.5 & - & - & - \\
\hline $8-2,25-26$ & 14.3 & - & - & 3.1 & 2.4 & - & - & - & - & 4.9 & 1.5 & - & - & - & 43.8 & - & - & 0.9 & - & 0.6 & - & - & - & - & - & - & - \\
\hline $8-2,26,0-27,5$ & 16.3 & - & - & 2.9 & 1.9 & - & - & - & - & 1.9 & 1.7 & - & - & - & 43.6 & 0.2 & 19.9 & 1.1 & - & 0.8 & - & - & - & - & 0.6 & - & - \\
\hline $8-2,27.5-28.0$ & 5.9 & - & - & 0.8 & 1.8 & - & - & - & - & 1.0 & 1.4 & - & - & - & 48.4 & 0.4 & 24.4 & 1.2 & - & 3.8 & - & - & - & 0.4 & - & - & - \\
\hline $8-2,29.0-30.5$ & 7.7 & - & - & 1.9 & 1.7 & - & - & - & - & 3.1 & 0.8 & - & - & - & 46.0 & 0.3 & 24.5 & 1.9 & - & 1.9 & - & - & - & 0.3 & 0.6 & - & - \\
\hline $8-2,30.5-32.5$ & 9.0 & - & - & 1.5 & 2.7 & - & - & - & - & 0.8 & 1.5 & - & - & - & 39.2 & 0.4 & 29.0 & 1.5 & - & 0.8 & - & - & - & 0.4 & 0.4 & - & - \\
\hline $8-2,32.5-33.5$ & 8.9 & - & 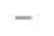 & 1.6 & 5.4 & - & - & - & - & - & 0.7 & - & - & - & 41.8 & - & 22.9 & 2.0 & - & 1.3 & - & - & - & 0.2 & - & - & - \\
\hline $8-2,33.5-34.5$ & 11.0 & - & - & - & 5.4 & - & - & - & - & 2.1 & 1.2 & - & - & - & 39.6 & 0.2 & 21.0 & 2.6 & - & 1.6 & - & - & - & 0.5 & 0.5 & - & - \\
\hline $8-2,34.5-35.5$ & 4.5 & - & - & 3.4 & 2.9 & - & - & - & - & 0.8 & 0.8 & 0.3 & - & - & 44.3 & - & 21.2 & 2.1 & - & 1.0 & - & - & - & 0.5 & - & - & - \\
\hline $8-2,35.5-37.0$ & 9.2 & - & - & 5.4 & 2.5 & - & - & - & - & 0.8 & 1.5 & 0.2 & - & - & 40.6 & - & 20.3 & 0.8 & - & 1.4 & - & - & - & 0.6 & 0.6 & - & - \\
\hline $8-2,37.0-39.0$ & 7.5 & - & - & 1.5 & 3.1 & - & - & - & - & 1.0 & 1.8 & - & - & - & 53.3 & - & 23.3 & 1.0 & - & 1.0 & - & - & - & 0.3 & 0.5 & - & - \\
\hline $8-2,39.0-410$ & 5.1 & - & - & 1.8 & 2.8 & - & - & - & - & 2.8 & 0.8 & 0.3 & - & - & 46.7 & 0.3 & 17.5 & 2.3 & - & 1.5 & - & - & - & - & 0.5 & 0.3 & - \\
\hline $8-2,41.0-42.0$ & 12.7 & - & - & 3.1 & 14.9 & - & - & - & - & 1.7 & 0.3 & - & - & - & 37.3 & - & 20.9 & 1.4 & - & 0.8 & - & - & - & 0.3 & 0.3 & - & - \\
\hline $8-2,42.0-44.0+$ & 5 & - & - & 3 & - & - & - & - & - & - & 1 & - & - & - & 12 & - & 11 & - & - & - & - & - & - & 3 & - & - & - \\
\hline $8-2,119-128$ & 16.0 & - & r & 2.8 & 0.6 & - & - & - & - & 1.1 & - & - & - & - & 45.6 & 1.1 & 21.0 & 1.7 & - & - & - & - & - & 0.6 & 1.1 & 0.6 & \\
\hline $8-3,27-35$ & 25.3 & - & - & 1.5 & 21.4 & - & - & - & - & 2.3 & - & - & - & - & 15.7 & - & 14.2 & 9.5 & - & - & - & - & - & 2.3 & - & - & - \\
\hline $9-3,90-98$ & 6.9 & - & - & 2.5 & 35.0 & - & - & - & - & 3.0 & - & - & - & - & 36.5 & - & 8.6 & 1.4 & - & - & - & - & - & 0.3 & - & - & - \\
\hline $10-1,35-39$ & 17.7 & - & - & 5.1 & 20.6 & - & - & - & - & 4.6 & - & - & - & - & 26.8 & 0.6 & 13.7 & 1.7 & - & - & - & - & - & 0.6 & 0.6 & - & - \\
\hline
\end{tabular}


Site 322 . At Site 323 the heavy subfraction is composed primarily of hornblende and epidote and the content of monoclinic pyroxenes rarely exceeds $10 \%$.

The content of magnetite (separate determination) and other opaque minerals is greater here than in the sediments from Site 322. Garnet, sphene, zircon, and apatite, which at Site 322 were found only as single grains, occur in measurable proportions at Site 323 . Among the minerals of the light subfraction, the content of quartz, feldspar, and opaline biogenic material is much greater than at Site 322. However, in the lower Miocene (Core 10, Sections 2 and 3) a layer occurs that contains a mineral suite in the coarse aleuritic fraction which is similar to those found in the Neogene sediments at Site 322.

In the Paleogene sediments, the content of clastic minerals in the coarse aleuritic fraction noticeably decreases relative to the overlying sediment, whereas the occurrence of authigenic minerals (hydrous ferric oxide and manganese) increases, as do barite and zeolites in the Paleocene and Maestrichtian sediments. The content of fish debris increases sharply and single interlayers rich in calcitic biogenic remains are present. At Site 323 barite constitutes nearly $74 \%$ of the heavy, coarse aleuritic subfraction from sediments above basalt.

\section{Sites 324 and 325}

Drilling at Sites 324 yielded sediments of PliocenePleistocene age. The mineral suites of the coarse aleuritic fractions are similar to those encountered in the upper part of the sequence at Site 323. The mineralogical make-up of the coarse aleuritic fraction of Site 324 is also similar to that of the upper part of the Pliocene-Pleistocene sequence at Site 325. In the Miocene, the mineralogy is similar to that of the Neogene sediments of Site 322 .

From analysis of the data given in Tables 11-14 we were able to distinguish at least three major mineral suites. The boundaries between them are not always sharp and consequently we could also have recognized transitional suites. For the purpose of this study, however, we restricted ourselves to only the principal suites (Table 15). The first two suites differ in their relative abundances of clastic minerals, which probably reflect compositions of the source rocks. The third suite is characterized by a distinct increase of authigenic minerals and fish debris and by a decrease in clastic minerals.

\section{DISCUSSION}

The mineralogical composition of sediments at Leg 35 sites is related to the geological history of the western part of Antarctica. During Mesozoic and Cenozoic time, the time during which the most ancient rocks of the area studied were being formed, there existed, instead of Antarctica, a vast orogenically arched uplift which in the Paleogene was subjected to intensive denudation (Grikurov, 1973). However, these processes are not reflected in the composition of the Maestrichtian and Paleogene sediments which were, on the contrary, formed under conditions of a minimal influx of terrigenous material. We believe that the clastic sediments (greater than $0.5 \mathrm{~mm}$ ) which were being predominantly transported by bottom currents were instead trapped in a deep trough which existed at that time. There was no sediment transport by ice-rafting, since glaciation of Antarctica did not begin until the Neogene. With the onset of the Paleogene, rift valleys formed in the central anticlinoriums and these retained most of the clastic particles. During Eocene-Oligocene time, the earlier sediments which had been formed under typical pelagic conditions were subjected to alteration by hydrothermal solutions. The solutions rose along fractures in the basaltic layer, diffused through the sediment, and ultimately reached the sea floor. Here, under benthonic conditions they caused the formation of a lithologically distinctive sediment. Initially, the chemical elements introduced by the hydrothermal solutions were subjected to differentiation as they moved through the sediments. This is indicated by their chemical composition and is discussed in greater detail elsewhere in this volume. The process is reflected in the mineral composition of the coarse aleuritic fraction by the formation of barite in the lowermost strata and enrichment of the sediments by free hydrous ferric oxide and manganese in the upper strata. At the Paleogene-Neogene boundary the depositional environment abruptly changed, with a substantial influx of terrigenous sedimentary material. This resulted from active block faulting of the Antarctic Andes following the intensive denudation and leveling of the late Mesozoic arched uplift. The block faulting also produced the rather dissected topography. Apparently, the Antarctic shelf in the Pacific sector was formed at the same time. Mineral suite $I$ is always present in the coarse aleuritic fraction of sediments deposited during this time. In the middle Miocene at Site 323 and Pliocene at Site 325 , suite I was replaced by suite II and at Site 322, suite I has occurred in sediments to the present time. This change of mineral suites in the clastic fraction is doubtless due to variations in the composition of the source rocks of western Antarctica. Presumably, mineral suite I was formed during the denudation of rocks of the Canyon Series, whereas mineral suite II incorporates rocks of the Canyon as well as the Scotia series.

Our study of the petrographic composition of the interbedded gravel layers (Site 324, Core 4 and Site 325, Core 2, Section 1), which are characteristic of suite II (Table 16), seemingly confirms our beliefs regarding the composition of source rocks on the Antarctic continent. The change in the mineral suites occurs earlier in the western part of the investigated area than in the eastern part, and at Site 322 deposition of suite I has been maintained to the present. This is indicative of the diverse denudation occurring on various parts of the Antarctic Coast or variations in the transportation complex from continent to ocean basin.

\section{REFERENCES}

Grikurov, G.A., 1973. Geology of Antarctic Peninsula: Moscow (Moscow Science Publishing Co.).

Lisitzin, A.P., Serova, V.V., et al., 1969. Geochemical, Mineralogical, and Paleontological Studies. In Fischer, A.G., Heezen, B.C., et al., Initial Reports of the Deep Sea Drilling Project, Volume 6: Washington (U.S. Government Printing Office), p. 829-960. 
TABLE 11B

Distribution of Minerals in the Heavy and Light Fraction

of Leg 35 Sediments

\begin{tabular}{|c|c|c|c|c|c|c|c|c|c|c|c|c|c|c|c|c|c|c|c|c|c|c|c|c|c|c|c|}
\hline $\begin{array}{c}\text { Sample } \\
\text { (Interval in } \mathrm{cm} \text { ) }\end{array}$ & 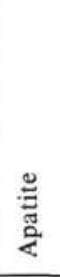 & 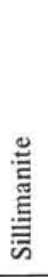 & 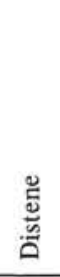 & 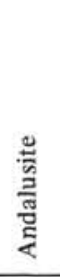 & $\begin{array}{l}\text { E } \\
\text { 总 } \\
\text { 范 }\end{array}$ & 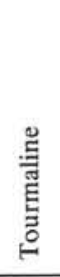 & 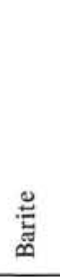 & 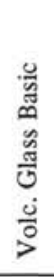 & 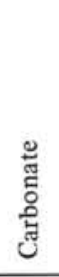 & $\frac{\stackrel{\Xi}{\Xi}}{\frac{0}{\circ}}$ & 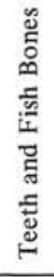 & 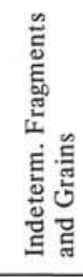 & 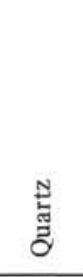 & 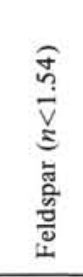 & 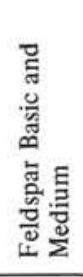 & 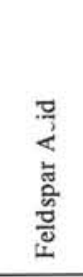 & 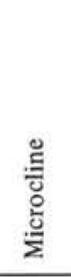 & $\frac{\stackrel{\Xi}{\tilde{\sigma}}}{\text { है }}$ & : & 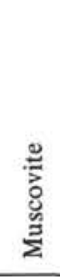 & 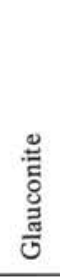 & 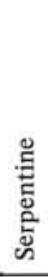 & 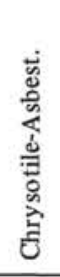 & 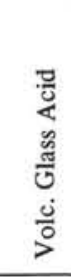 & 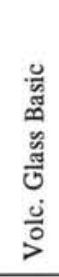 & $\begin{array}{l}\frac{\tilde{5}}{<} \\
\frac{\dot{j}}{0} \\
>\end{array}$ & 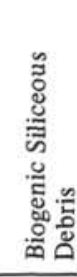 \\
\hline \multicolumn{28}{|l|}{ Site 322} \\
\hline $2-2,113-116$ & 0.4 & - & - & - & - & - & - & - & 0.4 & - & - & 1.8 & 13.6 & 11.7 & 7.2 & 4.5 & - & 0.3 & - & 0.3 & - & - & - & 1.0 & 1.0 & 7.2 & 1.0 \\
\hline $3-1,18-20$ & - & - & - & - & - & - & - & - & - & - & - & 5.0 & 10.9 & 5.8 & 8.2 & 6.0 & - & 0.7 & - & - & - & - & - & 0.2 & 0.7 & 6.3 & 2.5 \\
\hline $4-1,65-67$ & 0.3 & - & 0.3 & - & - & _- & 0.7 & - & 0.3 & - & - & 1.4 & 19.3 & 4.5 & 10.6 & 6.3 & - & 0.3 & - & - & - & - & - & - & - & 8.7 & - \\
\hline $4-2,57-60$ & 0.2 & - & 0.2 & - & - & - & 0.2 & - & - & - & - & 6.6 & 13.2 & 6.6 & 10.8 & 9.1 & - & 0.8 & 0.2 & - & - & - & - & 3.7 & 0.8 & 17.4 & 0.2 \\
\hline $5-1,81-85$ & - & - & - & - & - & - & - & - & - & - & - & - & 6.8 & 4.1 & 5.5 & 4.0 & - & - & - & - & - & - & - & 0.8 & 0.5 & 15.2 & 51.8 \\
\hline $6-1,30-33$ & 0.3 & - & - & - & - & - & 1.7 & - & 0.7 & - & - & 5.8 & 10.0 & 2.3 & 8.3 & 2.8 & - & 0.3 & - & - & - & - & - & 0.3 & 0.5 & 6.2 & 0.8 \\
\hline $9-2,143-146$ & 0.3 & - & - & - & - & - & 0.3 & - & - & - & - & 7.8 & 8.5 & 5.0 & 6.8 & 6.1 & - & 0.5 & - & - & - & - & - & 1.7 & 2.2 & 25.4 & 0.2 \\
\hline $10-1,101-105$ & 0.5 & - & - & - & - & - & - & - & 0.3 & - & - & 16.7 & 17.5 & 9.5 & 19.2 & 6.2 & - & - & - & - & - & - & - & 0.9 & 1.8 & 21.8 & - \\
\hline $10-2,43-46$ & 4 & - & - & - & - & 0.2 & - & - & 0.4 & - & - & 8.7 & 15.8 & 5.5 & 13.4 & 5.2 & - & 0.3 & - & - & - & - & - & - & 1.3 & 28.4 & - \\
\hline $11-1,42-46$ & 0.6 & - & - & - & - & - & - & - & - & - & - & 10.8 & 17.5 & 5.2 & 14.2 & 12.3 & - & - & 0.3 & - & - & - & - & - & - & - & - \\
\hline $11-2,45-49$ & 0.6 & - & - & - & - & - & - & - & - & - & - & 5.5 & 22.7 & 4.8 & 14.3 & 5.4 & - & 0.3 & - & - & - & - & - & - & - & 7.8 & - \\
\hline $11-3,24-32$ & 0.4 & - & - & - & - & - & - & - & 0.2 & - & - & 11.1 & 22.2 & 7.4 & 13.8 & 11.3 & - & - & - & - & - & - & - & - & - & - & - \\
\hline $11-4,17-24$ & 0.5 & - & - & - & - & - & - & - & 0.2 & - & - & 6.3 & 17.8 & 11.5 & 13.6 & 6.6 & - & 0.3 & - & - & - & - & - & - & - & - & - \\
\hline $11-5,36-40$ & 0.2 & - & - & - & - & - & - & - & - & - & - & - & 11.1 & 3.4 & 8.3 & 7.1 & - & - & 0.3 & 0.3 & - & - & - & 0.6 & - & 1.4 & 0.3 \\
\hline $11-6,109-115$ & 0.7 & - & - & - & - & 0.3 & - & - & - & - & - & - & 2.2 & 0.4 & 1.5 & 1.1 & - & 0.2 & - & - & - & - & - & 0.6 & - & 1.1 & - \\
\hline \multicolumn{28}{|l|}{ Site 323} \\
\hline $1-1,50-60$ & 4.8 & - & - & - & - & - & - & - & - & - & - & 4.4 & 39.3 & 24.6 & 1.4 & 1.8 & - & 1.1 & 0.4 & - & 0.4 & - & - & 0.4 & - & - & - \\
\hline $1-1,140$ - & & - & - & - & - & - & - & - & - & - & - & 6.0 & 40.8 & 24.6 & 0.4 & 4. & 0.4 & - & & - & - & - & & - & - & - & 0.9 \\
\hline $1-2,34-43$ & 2.1 & - & - & 0.4 & - & - & - & - & - & - & - & 6.4 & 29.5 & 17.2 & 0.3 & 4.6 & - & - & 0.3 & - & 0.3 & - & - & - & - & - & 0.3 \\
\hline $1-3,21-22$ & 1.5 & - & - & - & - & - & - & - & - & - & - & 7.7 & 33.2 & 21.3 & 0.4 & 1.7 & 0.4 & - & - & - & - & - & - & - & - & - & 0.4 \\
\hline $1-3,78-88$ & 1.0 & - & - & - & - & - & - & - & - & - & - & 4.0 & 22.7 & 12.4 & 1.9 & 3.9 & - & - & - & - & 0.4 & - & - & - & - & - & - \\
\hline $1-4,118-127$ & - & - & - & - & - & - & - & - & - & - & - & 3.0 & 23.8 & 6.9 & 1.6 & 2.0 & - & - & - & - & - & - & - & - & 0.4 & - & 17.2 \\
\hline $2-1,128-130$ & - & - & - & 1.0 & - & - & - & - & - & - & - & - & 11.7 & 8.3 & 0 . & 4.0 & - & 0.9 & - & - & - & - & - & - & 0.4 & - & 1.7 \\
\hline $3-1,88-94$ & 2.5 & - & - & - & - & - & - & - & - & - & - & 3.7 & 31.5 & 15.8 & 2.7 & 1.2 & - & - & - & - & - & - & - & 0.4 & 0.8 & - & 1.5 \\
\hline $3-2,14-20$ & 2.8 & - & - & - & - & - & - & - & - & - & - & 5.8 & 32.8 & 18.0 & 2.2 & 6.4 & - & 0.4 & - & - & 0.4 & - & - & - & - & - & 0.4 \\
\hline $3-2,61-70$ & 0.8 & - & - & - & - & - & - & - & - & - & - & - & 34.6 & 34.5 & 18.9 & 1.8 & 4.7 & 0.4 & 0.4 & - & - & - & - & - & - & 0.4 & - \\
\hline $5-1,145-176$ & - & - & - & - & - & - & - & - & - & - & - & 24.7 & 21.8 & 8.1 & 0.5 & 1.8 & - & - & - & - & - & - & - & - & - & - & 1.8 \\
\hline $6-1,100-110$ & - & - & - & - & - & - & - & - & - & - & - & 3 & 7.1 & 1.3 & 1.7 & 0. & - & 0.7 & - & - & - & - & - & 36.5 & 1.0 & - & - \\
\hline $6-1,140-142$ & - & - & - & _- & - & - & - & - & - & - & - & - & 11.0 & 1.6 & 0.8 & 1.1 & - & - & - & - & - & - & - & 0.5 & - & - & 1.1 \\
\hline $7-1,141-143$ & - & - & - & _- & - & _- & - & - & _- & - & - & 3.8 & 13.2 & 0.3 & 0.2 & 1. & - & - & - & - & - & - & - & 0.0 & - & - & 3.8 \\
\hline $7-2,105-110$ & - & - & - & - & - & _- & - & - & - & - & - & - & 13.9 & 2.8 & - & 1.8 & - & - & - & - & - & - & - & 25.4 & - & - & 6.1 \\
\hline $7-3,18-24$ & - & - & - & - & - & - & - & - & - & - & - & 2 & 10.1 & 0.5 & 0.5 & 1.4 & - & - & - & - & - & - & - & 5.5 & - & - & 3.2 \\
\hline $10-2,26-30$ & 0.2 & - & - & - & - & - & - & - & - & - & - & 6.1 & 11.8 & 4.6 & 19.7 & 0.4 & - & - & - & - & - & - & - & - & - & - & 0.4 \\
\hline $10-3,99-102$ & - & - & - & - & - & - & - & - & - & - & - & 11.2 & 19.2 & 5.3 & 31.2 & 1.9 & - & - & - & - & - & - & - & - & - & - & 0.4 \\
\hline $10-3,106-114$ & - & - & - & - & - & - & - & - & - & - & - & 5.3 & 9.1 & 1.2 & 6.3 & 0.3 & - & - & - & - & - & - & - & 0.3 & 0.3 & - & 8.4 \\
\hline $11-1,22-32$ & - & - & - & - & - & - & - & - & - & - & - & 26.3 & 37.8 & 17.4 & 0.9 & 1.4 & - & - & - & - & - & - & - & - & - & - & 7.7 \\
\hline $11-2,137-148$ & - & - & - & - & - & - & - & - & - & 23.8 & - & - & 18.9 & 7.8 & 2.3 & 4.2 & - & 0.9 & - & - & - & - & - & 0.9 & - & - & 0.9 \\
\hline $12-2,9-18$ & - & - & - & - & - & - & - & - & - & - & - & 26.1 & 39.5 & 18.3 & - & 0.7 & 0.2 & - & - & - & - & - & - & 0.2 & - & - & 2.1 \\
\hline $12-2,73-74$ & - & - & - & - & - & - & - & - & - & - & - & - & 47.5 & 23.1 & 1.9 & - & - & - & - & - & - & - & - & 0.5 & - & - & 0.5 \\
\hline $12-2,100-104$ & - & - & - & - & - & - & - & - & - & - & - & - & 40.4 & 18.2 & 0.2 & 1.5 & - & - & - & - & - & - & - & 0.2 & - & - & - \\
\hline $13-5,63-70$ & - & - & - & - & - & - & - & - & - & - & - & - & 21.0 & 4.8 & - & 0.5 & - & - & - & - & - & - & - & - & - & - & 0.6 \\
\hline $13-5,106-115$ & - & - & - & - & - & - & - & - & - & - & - & - & 30.0 & 6.3 & 0.3 & - & 0.3 & - & - & 0.5 & - & - & - & 0.3 & - & - & 2.8 \\
\hline $13-6,145-150$ & 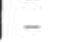 & - & - & _- & - & - & - & - & - & - & - & - & 14.7 & 5.7 & 0.5 & 1.0 & - & 0.5 & - & - & - & - & - & 35.0 & - & - & 1.0 \\
\hline
\end{tabular}




\begin{tabular}{|c|c|c|c|c|c|c|c|c|c|c|c|c|c|c|c|c|c|c|c|c|c|c|c|c|c|c|c|}
\hline $14-2,1-8$ & - & - & - & - & - & - & - & - & - & - & - & - & 21.0 & 19.2 & 0.3 & 1.0 & - & - & - & - & - & - & - & - & - & - & - \\
\hline $14-2,65-73$ & - & - & - & - & - & - & - & - & - & - & - & - & 20.9 & 5.7 & - & - & 0.3 & - & - & - & - & - & - & - & - & - & - \\
\hline $14-2,128-135$ & - & - & - & - & - & - & - & - & - & - & - & - & 7.7 & 2.5 & 0.6 & 0.3 & - & - & - & - & - & - & - & 0.3 & - & - & - \\
\hline $15-1,52-60$ & - & - & - & - & - & - & - & - & - & - & - & - & 0.8 & - & - & - & - & - & - & - & - & - & - & - & - & - & - \\
\hline $15-2,91-100$ & - & - & - & - & - & - & - & - & - & - & - & - & 3.4 & 1.5 & - & 0.8 & - & - & - & - & - & - & - & - & - & - & 0.4 \\
\hline $15-3,29-36$ & - & - & - & - & - & - & - & - & - & - & - & - & 3.3 & 0.4 & - & 0.7 & - & - & - & - & - & - & - & - & - & - & 0.4 \\
\hline $15-5,89-92$ & - & - & - & - & - & - & - & - & - & - & - & - & 13.8 & 3.5 & 0.4 & 1.8 & 0.4 & - & - & - & - & - & - & 1.3 & - & - & 6.7 \\
\hline $14-6,19-26$ & - & - & - & - & - & - & - & - & - & - & - & - & 3.3 & 0.8 & 0.8 & - & - & 0.8 & - & - & - & - & - & 0.4 & - & - & 3.3 \\
\hline $16-1,57-62$ & - & - & - & - & - & - & - & 0.4 & - & - & - & 1.3 & 1.7 & 0.3 & - & 0.3 & - & - & - & - & - & - & - & 1.0 & - & - & 8.6 \\
\hline $16-3,32-37$ & - & - & - & - & - & - & - & - & - & - & - & 3.7 & 7.3 & - & 0.8 & - & - & 0.4 & - & - & - & - & - & 25.9 & - & - & 0.8 \\
\hline $16-4,83-93$ & - & - & - & - & - & - & 73.8 & - & - & - & - & 1.6 & 1.8 & 0.9 & - & 0.4 & - & 0.5 & - & - & - & - & - & 8.0 & - & - & - \\
\hline $18-2,80-86$ & - & - & - & - & - & - & 23 & - & - & - & - & - & 0.8 & 0.4 & 0.8 & - & - & - & - & - & - & - & - & 6.5 & - & - & 22.9 \\
\hline $18-3,130-139$ & - & - & - & - & - & - & 29 & - & - & 1 & - & 3 & - & - & - & - & - & - & - & - & - & - & - & 0.4 & - & - & 90.8 \\
\hline $18-4,115-124$ & - & - & - & - & - & - & 3.3 & - & - & - & - & - & - & - & 0.8 & - & - & 0.4 & - & - & - & - & - & 0.4 & - & - & - \\
\hline $18-5,14-20$ & - & - & - & - & - & - & - & - & - & - & - & 1.4 & 3.6 & 3.0 & - & 0.5 & - & - & - & - & - & - & - & - & - & - & - \\
\hline $18-5,65-73$ & - & - & - & - & - & - & 1.0 & - & - & - & - & - & 1.2 & - & 0.8 & - & - & - & - & - & - & - & - & 0.8 & - & - & - \\
\hline $18-5,133-143$ & - & - & - & - & - & - & - & - & - & - & - & - & 5.3 & 3.0 & - & 0.6 & - & - & - & - & - & - & - & - & - & - & 3.3 \\
\hline $3, \mathrm{CC}$ & 1.5 & - & - & - & - & - & - & - & - & - & - & 4.9 & 48.5 & 13.5 & - & 0.7 & 0.7 & - & - & 0.4 & 0.7 & - & - & - & - & - & - \\
\hline \multicolumn{28}{|l|}{ Site 324} \\
\hline $1-2,126-131$ & - & - & - & - & - & - & - & - & - & - & - & 15 & 33.6 & 21.8 & 0.7 & 0.7 & 0.3 & 1.0 & - & - & 0.3 & - & - & 2.0 & - & - & 0.3 \\
\hline $1-4,125-131$ & - & - & - & - & - & - & - & - & - & - & - & 11.3 & 37.8 & 19.8 & 0.5 & 0.5 & - & - & - & - & 0.5 & - & - & 0.8 & - & - & 0.5 \\
\hline $1-6,31-38$ & 2.4 & - & - & - & - & - & - & - & - & - & - & 8.1 & 36.7 & 26.0 & 1.4 & 1.7 & 0.3 & 0.7 & 0.7 & - & 0.7 & - & - & 0.3 & 0.3 & - & - \\
\hline $2-1,83-92$ & - & - & - & - & - & - & - & - & - & - & - & 30.7 & 30.9 & 17.3 & 2.5 & 1.8 & - & - & - & - & - & - & - & - & - & - & 0.9 \\
\hline $2-2,61-70$ & - & - & - & - & - & - & - & - & - & - & - & 29.8 & 25.4 & 16.4 & 0.9 & 1.1 & 0.3 & 1.7 & - & - & - & - & - & - & - & - & 0.9 \\
\hline $2-3,52-61$ & - & _ & - & - & - & - & - & - & _- & - & - & 28.4 & 31.4 & 12.0 & 4.2 & 3.9 & 0.3 & - & - & - & - & - & - & - & - & - & - \\
\hline $2-4,27-36$ & - & - & - & - & - & - & - & - & - & - & - & 18.7 & 29.5 & 35.2 & 1.3 & 0.7 & 0.4 & - & - & - & - & - & - & - & - & - & - \\
\hline $2-5,35-45$ & - & - & - & - & - & - & - & - & - & - & - & 7.7 & 35.8 & 39.7 & 0.3 & 1.8 & - & 1.8 & 0.8 & - & - & - & - & 1.8 & - & - & - \\
\hline $2-6,95-104$ & - & - & - & - & - & - & - & - & - & - & - & 24.0 & 38.2 & 30.8 & - & 0.6 & - & - & - & - & 0.6 & - & - & - & - & - & 0.6 \\
\hline $3-1,137-146$ & - & - & - & - & - & - & - & - & - & - & - & 21.5 & 26.6 & 14.0 & 1.4 & 1.4 & - & 0.4 & - & - & - & - & - & - & - & - & - \\
\hline $3-2,136-145$ & - & - & - & - & - & - & - & - & - & - & - & 18.8 & 43.0 & 18.3 & 1.0 & 0.5 & - & - & - & - & - & - & - & - & - & - & - \\
\hline $3-3,67-77$ & - & - & - & - & - & - & - & - & - & - & - & 15.0 & 18.6 & 4.5 & 2.8 & 0.8 & - & - & - & - & - & - & -. & 0.3 & - & - & 3.1 \\
\hline $3-4,47-57$ & - & - & - & - & - & - & - & - & - & - & - & 4.2 & 2.1 & 1.4 & 1.1 & 0.4 & - & - & - & - & - & - & - & 0.7 & - & - & - \\
\hline $3-5,99-100$ & - & - & - & - & - & - & - & - & - & - & - & - & 4.5 & 4.0 & 0.7 & 1.2 & - & - & - & - & - & - & - & - & - & - & - \\
\hline $4-2,113-123$ & - & - & - & - & - & - & - & - & - & - & - & 22.5 & 34.2 & 15.7 & 1.1 & 1.1 & - & - & - & - & - & - & - & 0.5 & - & - & - \\
\hline $4-3,30-40$ & - & - & - & - & - & - & - & - & - & - & - & 35.0 & 39.2 & 20.5 & - & 1.3 & _- & - & - & - & 0.9 & - & - & 0.5 & - & - & - \\
\hline $4-4,54-64$ & _- & - & - & - & - & - & - & - & - & - & - & 12.4 & 32.4 & 21.3 & 1.5 & 0.8 & _- & - & - & - & 0.4 & - & - & 0.8 & 0.8 & - & 0.8 \\
\hline $5-2,66-83$ & _ & - & - & - & - & - & - & - & - & - & - & - & 35.6 & 24.6 & 0.6 & 1.0 & - & - & - & - & 0.2 & - & - & - & - & - & 0.6 \\
\hline $5-3,25-35$ & - & - & - & - & - & - & - & - & - & - & - & - & 0.6 & - & - & - & - & - & - & - & - & - & - & - & - & - & - \\
\hline $7-1,69-78$ & - & - & - & - & - & - & - & - & - & - & - & - & 16 & 11 & - & 2 & 1 & - & - & - & - & - & - & - & - & - & 1 \\
\hline $7-3,110-120$ & - & - & - & - & - & - & - & - & - & - & - & 14.8 & 35.0 & 10.0 & 0.3 & - & - & - & - & 0.3 & - & - & - & 0.3 & - & - & - \\
\hline $7-6,119-129$ & - & - & - & - & - & - & - & - & - & - & - & - & 35.4 & 14.0 & 0.3 & - & - & - & - & - & 0.5 & - & - & 0.8 & - & - & 0.5 \\
\hline $8-3,61-71$ & - & - & - & - & - & - & - & - & - & - & - & 5.7 & 8.2 & 4.9 & - & 0.3 & - & - & - & - & - & - & - & 1.1 & - & - & 0.5 \\
\hline \multicolumn{28}{|l|}{ Site 325} \\
\hline $1-1,49-59$ & 0.3 & - & - & - & - & - & 0.3 & - & - & - & - & 7.6 & 15.2 & 11.0 & 3.7 & 4.6 & - & 0.5 & 0.5 & - & - & - & - & 0.5 & - & - & - \\
\hline $1-1,133-144$ & 0.5 & - & - & - & - & - & 0.5 & - & - & - & - & 7.9 & 10.6 & 3.9 & 1.6 & 1.2 & - & - & - & - & - & - & - & - & - & - & 0.4 \\
\hline $1-2,26-36$ & - & - & - & - & - & - & - & - & - & - & - & - & 22.6 & 3.8 & 4.7 & 2.8 & - & 1.4 & - & - & - & - & - & 0.9 & - & - & 3.8 \\
\hline $1-4,181-192$ & 0.2 & - & - & - & - & - & 0.7 & - & - & _- & - & 6.1 & 29.8 & 18.0 & 4.2 & 2.9 & - & 0.3 & - & - & - & - & - & - & - & - & 0.3 \\
\hline $2-2,39-49$ & 1.3 & - & - & - & - & - & - & - & - & - & - & 7.7 & 30.2 & 12.1 & 9.5 & 1.3 & - & 0.4 & - & - & - & - & - & 0.4 & - & - & 0.4 \\
\hline $3-2,11-24$ & 0.7 & _- & - & - & _ & - & - & - & _- & - & - & 7.3 & 29.0 & 6.8 & 3.6 & 4.1 & - & 0.9 & - & - & - & - & - & 0.5 & - & - & - \\
\hline $3-3,76-87$ & 0.7 & - & - & - & - & - & - & - & - & - & - & 11.5 & 30.2 & 15.2 & 13.0 & 0.9 & - & 1.8 & - & - & - & - & - & 0.4 & - & - & 0.9 \\
\hline $3-4,26-36$ & 0.5 & - & - & - & - & - & - & - & - & - & - & 6.3 & 22.0 & 10.4 & 7.8 & 2.2 & - & 2.6 & - & - & - & - & - & 0.4 & - & - & - \\
\hline $5-1,55-57$ & - & - & - & - & - & - & - & - & - & - & - & 4.9 & 17.7 & 2.4 & 18.1 & 7.6 & - & - & - & - & - & - & - & - & - & - & - \\
\hline $5, \mathrm{CC}$ & - & - & - & - & - & - & - & - & - & - & - & 5.8 & 14.3 & 5.4 & 23.4 & 3.7 & - & - & - & - & - & - & - & 2.9 & - & - & - \\
\hline
\end{tabular}


TABLE 11B - Continued

\begin{tabular}{|c|c|c|c|c|c|c|c|c|c|c|c|c|c|c|c|c|c|c|c|c|c|c|c|c|c|c|c|}
\hline $\begin{array}{c}\text { Sample } \\
\text { (Interval in cm) }\end{array}$ & 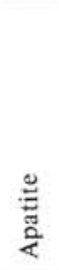 & 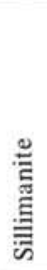 & $\frac{\dddot{\Xi}}{\frac{m}{0}}$ & 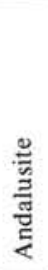 & ह & 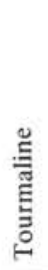 & 莺 & 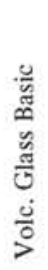 & 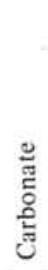 & $\frac{\stackrel{\mathscr{C}}{\Xi}}{\stackrel{\circ}{\circ}}$ & 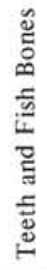 & 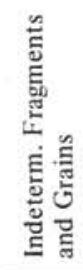 & $\begin{array}{l}\text { Ũ } \\
\text { בे }\end{array}$ & 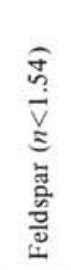 & 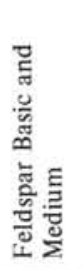 & 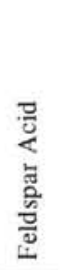 & 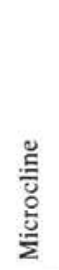 & 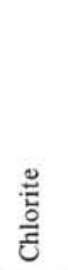 & 苛 & 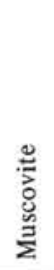 & 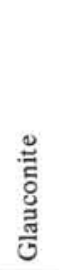 & 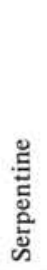 & 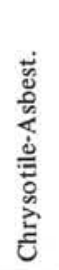 & 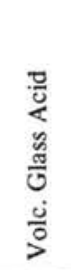 & 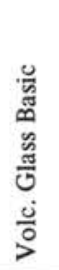 & $\begin{array}{l}\frac{5}{4} \\
\dot{0} \\
\frac{0}{0}\end{array}$ & 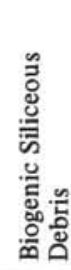 \\
\hline $6-1,145-150$ & - & - & - & - & - & - & - & - & - & - & - & 4.3 & 22.0 & 6.8 & 25.7 & 1.0 & - & - & - & - & - & - & - & 0.5 & - & - & 6.3 \\
\hline $7-1,131-134$ & - & - & - & - & - & - & - & - & - & - & - & 6.1 & 24.3 & 4.3 & 28.0 & 0.4 & - & - & - & - & - & - & - & - & - & - & - \\
\hline $7-2,138-142$ & - & - & - & - & - & - & - & - & - & - & - & 7.3 & 19.5 & 4.9 & 25.9 & 0.8 & - & - & - & - & - & - & - & 0.8 & - & - & 3.8 \\
\hline $8-1,45-49$ & - & - & - & - & - & - & - & - & - & - & - & 5.8 & 39.4 & 9.6 & 7.2 & 0.6 & - & 0.6 & _- & - & - & _- & - & 1.2 & - & _- & 31.2 \\
\hline $8-2,22.5-23.5$ & - & - & - & - & - & - & - & - & - & - & - & 18.5 & 18.0 & 1.7 & 37.0 & 0.6 & - & - & - & - & 0.6 & - & - & - & - & - & 0.6 \\
\hline $8-2,23.5-25.0$ & - & - & - & - & - & - & - & - & - & - & - & 14.1 & 12.1 & 10.4 & 25.5 & - & - & - & - & - & 0.4 & - & - & - & - & 11.3 & - \\
\hline $8-2,25-26$ & - & - & - & - & - & - & - & - & - & - & - & 5.2 & 17.5 & 5.6 & 25.0 & 0.4 & - & 0.4 & - & - & - & - & - & - & - & - & - \\
\hline $8-2,26.0-27.5$ & - & - & - & - & - & - & - & - & - & - & - & 9.1 & 10.2 & 3.8 & 33.0 & - & - & - & - & - & - & - & - & - & - & - & - \\
\hline $8-2,27.5-28.0$ & 0.2 & - & - & - & - & - & - & - & - & - & - & 15.3 & 11.9 & 2.5 & 33.7 & - & - & - & - & - & 0.4 & - & - & - & - & - & 48.5 \\
\hline $8-2,29.0-30.5$ & - & - & - & - & - & - & - & - & - & - & - & 9.3 & 7.6 & 4.9 & 32.6 & 0.8 & - & 2.3 & - & - & - & - & - & - & - & - & - \\
\hline $8-2,30.5-32.5$ & 0.2 & - & - & - & - & - & - & - & - & - & - & 12.6 & 11.6 & 4.9 & 24.3 & 0.4 & - & - & - & - & - & - & - & - & - & - & - \\
\hline $8-2,32.5-33.5$ & 0.5 & - & - & - & - & - & - & - & - & - & - & 14.7 & 13.8 & 1.4 & 35.4 & - & - & - & - & - & - & - & - & - & - & 21.5 & 0.9 \\
\hline $8-2,33.5-34.5$ & 0.2 & - & - & - & - & - & - & - & - & - & - & 14.0 & 13.2 & 4.9 & 29.5 & - & - & - & - & - & - & - & - & - & - & - & - \\
\hline $8-2,34.5-35.5$ & - & - & - & - & - & - & - & - & - & - & - & 18.2 & 5.0 & 6.7 & 26.4 & - & - & - & - & - & - & _ & - & - & - & 13.4 & - \\
\hline $8-2,35.5-37.0$ & 0.6 & - & - & - & - & - & - & - & - & - & - & 14.5 & 11.3 & 8.7 & 26.1 & 0.4 & - & - & - & - & - & _- & - & - & - & 11.3 & 0.4 \\
\hline $8-2,37.0-39.0$ & 0.3 & - & - & - & - & - & - & - & - & - & - & 5.4 & 11.7 & 3.8 & 30.4 & 1.1 & - & 0.4 & - & - & - & - & - & - & - & - & 0.8 \\
\hline $8-2,39.0-41.0$ & - & - & - & - & - & - & - & - & - & - & - & 17.3 & 20.9 & 1.8 & 27.5 & - & - & 0.4 & - & - & - & - & - & - & - & 11.7 & - \\
\hline $8-2,41.0-42.0$ & - & - & - & - & - & - & - & - & - & - & - & 6.3 & 4.6 & 4.2 & 20.2 & - & - & 1.1 & - & - & - & - & - & - & - & - & 2.3 \\
\hline $8-2,42.0-44.0$ & - & - & - & - & - & - & - & - & - & - & - & - & - & - & - & - & - & - & - & - & - & - & - & - & - & - & 78.9 \\
\hline $8-2,119-128$ & - & - & - & - & - & - & - & - & - & - & - & 7.8 & 17.1 & 2.8 & 21.9 & 0.9 & - & - & - & - & - & - & & & & - & \\
\hline $8-3,27-35$ & - & - & - & - & - & - & - & - & - & - & - & 7.1 & 23.3 & 4.3 & 20.2 & 2.1 & 0.4 & - & - & - & - & - & - & 0.9 & - & - & 20.2 \\
\hline $9-3,90-98$ & - & - & - & - & - & - & - & - & - & - & - & 5.0 & 10.5 & 3.8 & 24.2 & - & - & - & - & - & - & - & - & 1.4 & - & - & - \\
\hline $10-1,35-39$ & - & - & - & - & - & - & - & - & - & - & - & 8.0 & 5.5 & 1.2 & 6.3 & 0.8 & - & - & - & - & - & - & - & 0.8 & - & - & 14.8 \\
\hline
\end{tabular}


TABLE 11C

Distribution of Minerals in the Heavy and Light Fraction of Leg 35 Sediments

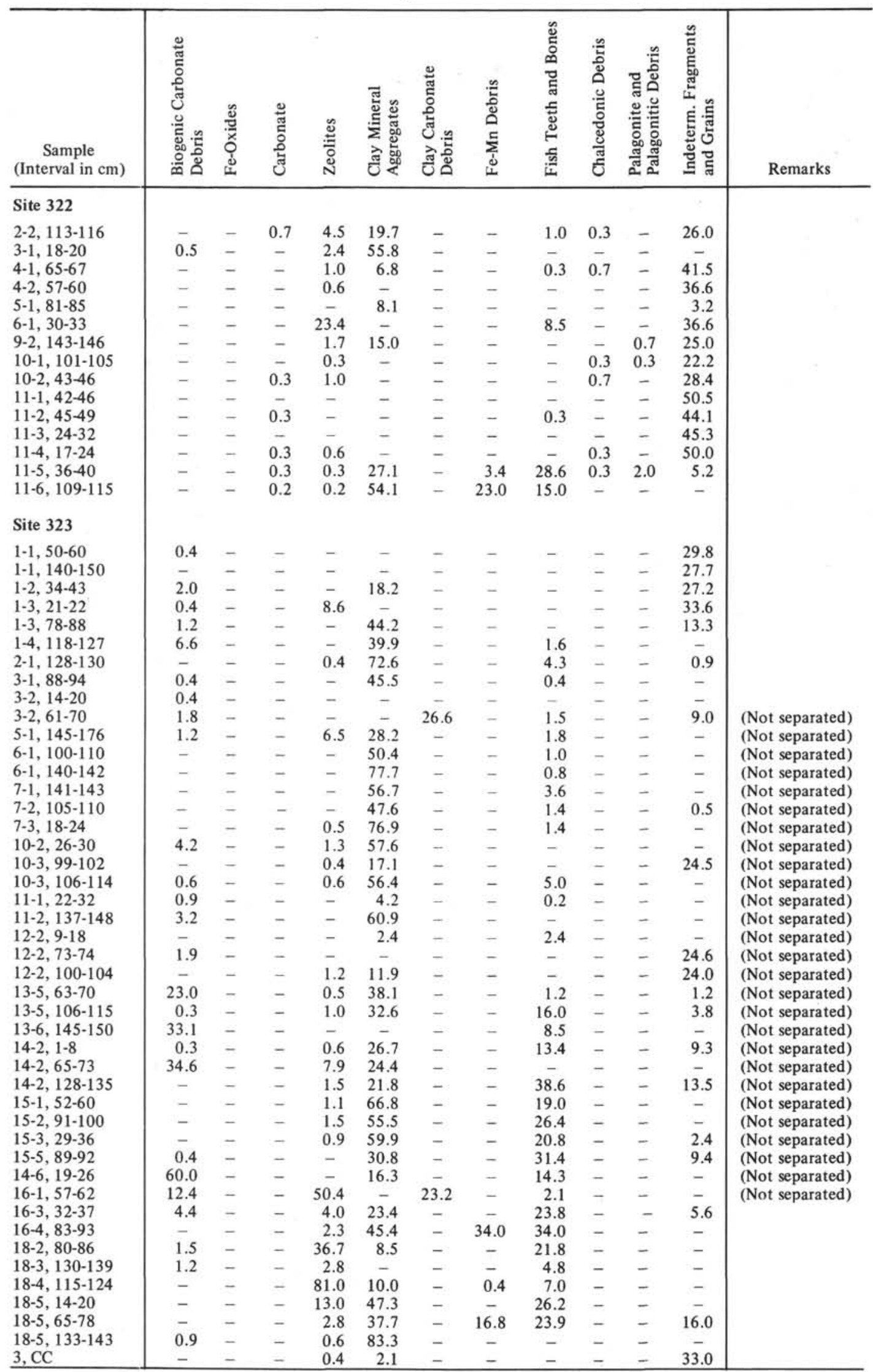


TABLE 11C - Continued

\begin{tabular}{|c|c|c|c|c|c|c|c|c|c|c|c|c|}
\hline $\begin{array}{c}\text { Sample } \\
\text { (Interval in } \mathrm{cm} \text { ) }\end{array}$ & 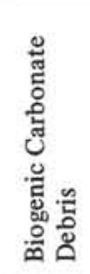 & 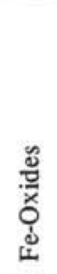 & 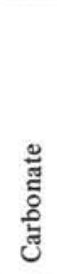 & $\begin{array}{l}\stackrel{\Xi}{0} \\
\stackrel{\Xi}{\Delta} \\
\text { N }\end{array}$ & 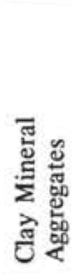 & 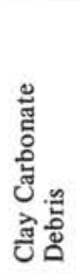 & $\begin{array}{l}\stackrel{n}{5} \\
0 \\
0 \\
\sum_{0}^{5} \\
0\end{array}$ & 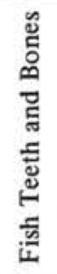 & 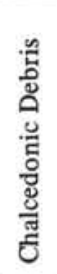 & 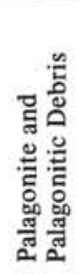 & 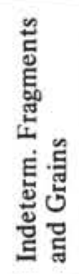 & Remarks \\
\hline \multicolumn{13}{|l|}{ Site 324} \\
\hline $1-2,126-131$ & - & - & - & - & - & - & - & 0.7 & - & - & 38.6 & \\
\hline $1-4,125-131$ & - & - & - & - & 17.4 & - & 3.3 & - & - & - & - & \\
\hline $1-6,31-38$ & 0.7 & - & - & - & - & - & - & - & - & - & 30.5 & \\
\hline $2-1,83-92$ & - & - & - & 0.6 & 9.6 & - & - & - & - & - & - & \\
\hline $2-2,61-70$ & - & - & - & - & 21.0 & - & - & 1.4 & - & - & - & \\
\hline $2-3,52-61$ & - & - & - & 0.6 & 8.6 & - & - & 0.8 & - & - & - & \\
\hline $2-4,27-36$ & - & - & - & 0.4 & 6.4 & - & - & - & - & - & - & \\
\hline $2-5,35-45$ & - & - & - & 1.5 & - & - & - & - & - & - & - & \\
\hline $2-6,95-104$ & 2.3 & - & - & - & - & - & - & - & - & - & - & \\
\hline $3-1,137-146$ & - & - & - & - & 4.2 & - & - & - & - & - & - & \\
\hline $3-2,136-145$ & - & - & - & - & 4.8 & - & - & - & - & - & - & \\
\hline $3-3,67-77$ & 0.3 & - & - & 42.3 & - & - & - & - & - & - & - & \\
\hline $3-4,47-57$ & 0.7 & - & - & - & - & - & - & 0.4 & - & - & - & \\
\hline $3-5,99-100$ & - & - & - & - & 85.0 & - & - & - & - & - & - & \\
\hline $4-2,113-123$ & - & - & 1.3 & - & 11.7 & - & - & - & - & - & - & \\
\hline $4-3,30-40$ & - & - & - & - & - & - & - & - & - & - & - & \\
\hline $4-4,54-64$ & 0.8 & - & - & - & 20.8 & - & - & - & - & - & - & \\
\hline $5-2,66-83$ & - & - & - & 4.9 & 3.9 & - & - & - & - & - & 21.5 & \\
\hline $5-3,25-35$ & - & - & - & 99.4 & - & - & - & - & - & - & - & \\
\hline $7-1,69-78$ & 1 & - & - & 44 & 3 & - & - & - & - & - & 6 & \\
\hline $7-3,110-120$ & - & - & - & 15.8 & 5.7 & - & - & - & - & - & - & \\
\hline $7-6,119-129$ & 3.3 & - & - & 9.6 & 2.2 & - & - & - & - & - & 7.7 & \\
\hline $8-3,61-71$ & 0.3 & - & - & 57.5 & - & - & - & - & - & - & - & \\
\hline \multicolumn{13}{|l|}{ Site 325} \\
\hline $1-1,49-59$ & 15.2 & - & - & - & 23.3 & - & - & - & - & - & 25.5 & \\
\hline $1-1,133-134$ & 21.4 & - & - & - & 52.6 & - & 0.8 & - & - & - & 6.7 & \\
\hline $1-2,26-36$ & 7.0 & - & - & - & 53.0 & - & - & - & - & - & - & \\
\hline $1-4,181-192$ & 2.9 & - & - & 0.3 & 16.1 & - & - & - & - & - & 25.2 & \\
\hline $2-2,39-49$ & 2.2 & - & - & - & 43.5 & - & - & - & - & - & - & \\
\hline $3-2,11-24$ & 1.4 & - & - & - & 53.7 & - & - & - & - & - & - & \\
\hline $3-3,76-87$ & 1.4 & - & - & - & 36.7 & - & - & - & - & - & - & \\
\hline $3-4,26-36$ & 1.7 & - & - & - & 52.9 & - & - & - & - & - & - & \\
\hline $5-1,55-57$ & - & - & - & 0.4 & 44.7 & - & - & - & - & - & 9.1 & \\
\hline $5, \mathrm{CC}$ & 0.4 & - & - & - & 40.3 & - & - & - & - & - & 9.6 & \\
\hline $6-1,145-150$ & 1.6 & - & - & - & 22.5 & - & - & - & - & - & 13.7 & \\
\hline $7-1,131-134$ & - & - & - & - & 33.2 & - & 2.6 & - & - & - & 6.4 & \\
\hline $7-2,138-142$ & 0.4 & - & - & 0.4 & 43.5 & - & - & - & - & - & - & \\
\hline $8-1,45-49$ & - & - & - & - & 10.2 & - & - & _- & - & - & - & \\
\hline $8-2,22.5-23.5$ & - & - & - & - & - & - & - & - & - & - & 41.5 & \\
\hline $8-2,23.5-25.0$ & - & - & - & - & - & - & - & - & - & - & 40.3 & \\
\hline $8-2,25-26$ & 0.4 & 0.8 & - & - & - & - & - & - & - & - & 49.9 & \\
\hline $8-2,26.0-27.5$ & - & - & - & - & - & - & - & - & - & - & 53.0 & \\
\hline $8-2,27.5-28.0$ & - & - & - & - & - & - & - & - & - & - & - & \\
\hline $8-2,29.0-30.5$ & 1.5 & - & - & - & - & - & - & - & - & - & 50.4 & \\
\hline $8-2,30.5-32.5$ & - & - & - & 6.7 & 4.2 & - & - & - & - & - & 47.9 & \\
\hline $8-2,32-5-33.5$ & 0.5 & - & - & 3.8 & - & - & - & - & - & - & 22.2 & \\
\hline $8-2,33.5-34.5$ & - & - & - & 9.4 & 12.4 & - & - & - & - & $\ldots$ & 22.5 & \\
\hline $8-2,34.5-35.5$ & - & _ & - & - & - & - & - & - & - & - & 48.5 & \\
\hline $8-2,35.5-37.0$ & - & - & - & - & - & - & - & - & - & - & 46.8 & \\
\hline $8-2,37.0-39.0$ & - & - & - & 13.6 & - & - & - & - & - & - & 38.2 & \\
\hline $8-2,39.0-41.0$ & - & - & - & - & - & - & - & - & -. & - & 37.7 & \\
\hline $8-2,41.0-42.0$ & 0.4 & - & _- & - & 14.5 & - & _. & $=$ & - & - & 27.9 & \\
\hline $8-2,42.0-44.0$ & 16.7 & - & & - & & - & -. & - & - & - & 0.6 & \\
\hline $8-2,119-128$ & 30.7 & - & - & 0.9 & 25.2 & - & - & - & - & - & - & \\
\hline $8-3,27-35$ & 5.2 & - & -.. & - & 23.2 & -- & - & - & - & & - & \\
\hline $9-3,90-98$ & 1.0 & - & - & & 40.5 & - & - & - & - & - & - & \\
\hline $10-1,35-39$ & 0.4 & - & - & - & 70.2 & - & & - & - & - & - & \\
\hline
\end{tabular}


TABLE 12

Distribution of Minerals in Light Fraction of Samples (Elutriated)

\begin{tabular}{|c|c|c|c|c|c|c|c|c|c|c|c|c|c|c|c|c|c|c|c|c|c|c|}
\hline $\begin{array}{c}\text { Sample } \\
\text { (Interval } \\
\text { in } \mathrm{cm} \text { ) }\end{array}$ & 롤 & 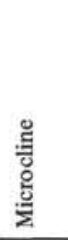 & 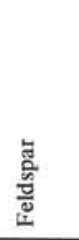 & 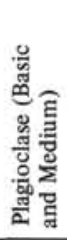 & 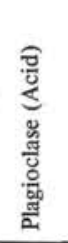 & 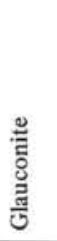 & : & 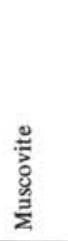 & $\begin{array}{l}\text { : } \\
\text { है }\end{array}$ & 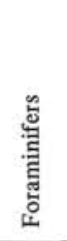 & 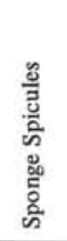 & 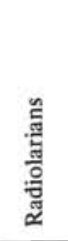 & $\begin{array}{l}\text { है } \\
\text { है } \\
\text { ă }\end{array}$ & 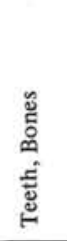 & 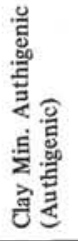 & 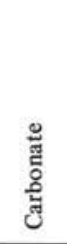 & 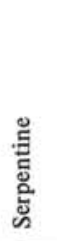 & 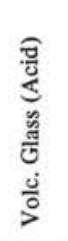 & 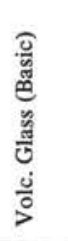 & 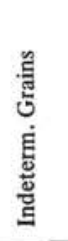 & 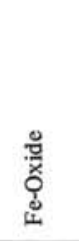 & 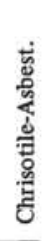 \\
\hline \multicolumn{23}{|l|}{ Site 322} \\
\hline $1, \mathrm{CC}$ & 27.6 & - & 11.1 & 12.9 & - & 1.7 & 1.2 & 1.2 & 2.3 & 6.4 & - & - & 1.7 & - & - & - & - & - & - & 31.6 & 2.3 & - \\
\hline $2, \mathrm{CC}$ & 14.7 & - & 8.3 & 14.1 & - & - & 0.6 & - & - & - & 2.6 & 3.2 & - & - & - & - & - & 3.2 & - & 53.3 & - & - \\
\hline $3, \mathrm{CC}$ & 16.5 & - & 8.5 & 19.4 & - & - & 0.8 & - & - & - & - & - & 2.0 & - & 29.4 & - & - & 1.6 & 2.0 & 19.8 & - & - \\
\hline $4, \mathrm{CC}$ & 13.5 & - & 6.7 & 12.3 & - & - & 2.5 & 0.6 & 1.8 & - & - & 1.2 & 0.6 & - & 19.6 & - & - & 4.9 & 3.1 & 33.2 & - & - \\
\hline $5, \mathrm{CC}$ & 10.6 & 0.7 & 7.0 & 12.7 & - & - & 1.4 & - & 1.4 & - & - & 2.8 & - & 12.0 & 23.2 & - & - & - & 1.4 & 26.8 & - & - \\
\hline $6, \mathrm{CC}$ & 15.4 & - & 4.7 & 15.4 & - & - & 0.9 & 0.5 & - & - & - & 1.9 & - & - & 16.4 & - & - & - & - & 42.9 & 1.9 & - \\
\hline $10, \mathrm{CC}$ & 17.5 & - & 10.5 & 18.5 & - & - & 0.5 & - & 0.5 & - & - & - & 0.5 & - & 18.5 & - & - & - & - & 32.0 & 1.5 & - \\
\hline $11, \mathrm{CC}$ & 14.5 & - & 6.2 & 17.6 & - & - & - & - & - & - & - & 1.0 & - & 3.6 & 11.9 & - & - & - & 2.1 & 32.2 & 10.9 & - \\
\hline \multicolumn{23}{|l|}{ Site 323} \\
\hline $1, \mathrm{CC}$ & 2.5 & - & - & 1.0 & - & - & - & - & 1.0 & - & - & 67.7 & - & - & 24.8 & - & - & - & - & 3.0 & - & - \\
\hline $2, \mathrm{CC}$ & 40.4 & - & 12.5 & 11.7 & - & - & 0.4 & - & 1.0 & - & - & 1.7 & - & - & 9.1 & - & - & 0.9 & - & 22.9 & 0.4 & - \\
\hline $3, \mathrm{CC}$ & 32.1 & - & 13.5 & 12.7 & 3.5 & - & - & - & 1.8 & - & 0.9 & 8.3 & 1.8 & - & - & - & 0.4 & 0.9 & 0.4 & 21.9 & 0.9 & 0.9 \\
\hline $4, C C$ & 17.4 & - & 14.5 & 11.0 & - & - & - & - & 1.8 & - & - & 9.3 & - & - & 10.5 & - & - & 0.6 & - & 35.5 & 1.2 & - \\
\hline $5, \mathrm{CC}$ & 22.9 & - & 13.8 & 15.4 & - & 0.5 & 4.3 & - & 1.1 & - & 10.1 & 5.9 & 0.5 & - & - & - & - & 0.5 & - & 20.2 & 4.8 & - \\
\hline $6, \mathrm{CC}$ & 38.0 & - & 10.1 & 5.1 & - & - & 4.3 & 0.6 & 1.3 & - & 1.3 & 11.4 & - & - & 9.5 & - & - & - & - & 22.7 & - & - \\
\hline $7, \mathrm{CC}$ & 40.6 & - & 10.0 & 12.0 & - & - & 0.5 & - & 1.9 & - & - & - & - & - & 9.6 & - & - & 1.0 & - & 24.4 & - & - \\
\hline $8-3$ & 2.9 & - & 2.9 & 3.4 & - & - & - & - & - & - & - & 5.7 & 3.4 & - & - & - & - & - & - & 81.7 & - & - \\
\hline $8, \mathrm{CC}$ & 36.9 & - & 17.2 & 9.6 & - & - & 1.5 & 1.0 & 0.5 & 2.5 & - & - & 1.0 & - & - & - & - & 0.5 & - & 27.3 & 2.0 & - \\
\hline $9, \mathrm{CC}$ & 22.9 & - & 8.8 & 5.3 & - & - & - & - & 1.2 & - & 0.6 & 14.7 & - & - & 13.5 & - & - & 1.8 & - & 31.2 & - & - \\
\hline $10, \mathrm{CC}$ & 0.8 & - & - & 0.4 & - & - & - & - & - & - & - & 0.8 & - & - & 89.6 & 4.4 & - & - & - & 4.0 & - & - \\
\hline $12, \mathrm{CC}$ & 8.8 & - & 0.8 & 1.3 & - & - & - & - & - & 3.8 & - & 0.4 & 0.8 & - & - & - & - & - & - & 1.7 & 82.4 & - \\
\hline $13, \mathrm{CC}$ & 4.5 & - & 4.9 & 6.5 & - & 0.4 & - & - & - & 53.1 & - & - & - & 6.5 & - & - & - & - & - & 19.6 & 4.5 & - \\
\hline $15, \mathrm{CC}$ & 9.6 & - & 1.5 & 1.5 & - & - & - & - & 1.5 & - & - & 8.9 & 0.7 & 24.4 & 17.1 & 31.1 & - & 2.2 & - & - & 1.5 & - \\
\hline $17, \mathrm{CC}$ & - & - & - & - & - & 1.2 & - & - & - & 83.7 & - & 1.7 & 4.7 & 8.1 & - & - & - & - & - & - & 0.6 & - \\
\hline \multicolumn{23}{|l|}{ Site 324} \\
\hline $1-2$ & 39.1 & 0.5 & 15.0 & 16.4 & 1.4 & - & - & - & 1.4 & - & - & - & - & - & 1.0 & - & - & 1.9 & 0.5 & 23.2 & -.. & - \\
\hline $1-3$ & & - & 7.3 & 10.4 & - & - & 1.2 & - & & - & - & 3.0 & - & - & 11.7 & - & - & 12.2 & 2.5 & 22 & - & - \\
\hline $3, \mathrm{CC}$ & 36.5 & - & 10.3 & 11.2 & - & 1.4 & - & - & 0.9 & - & - & 2.3 & - & - & 6.5 & - & - & 0.5 & - & 30.4 & - & - \\
\hline $4, C C$ & 28.9 & 0.4 & 12.2 & 14.6 & - & - & - & - & 1.7 & - & 1.3 & 0.4 & - & - & 11.2 & - & - & 1.7 & 0.8 & 20.4 & 6.4 & - \\
\hline $5, \mathrm{CC}$ & 36.3 & - & 18.3 & 9.4 & - & - & 1.1 & - & 1.7 & - & - & - & - & - & 5.5 & - & - & 1.1 & - & 24.4 & 2.2 & - \\
\hline $6, \mathrm{CC}$ & 29.6 & - & 7.2 & 13.8 & - & - & 0.7 & - & 2.0 & - & - & - & - & - & 25.0 & - & - & 2.6 & 0.7 & 18.4 & - & - \\
\hline $7, \mathrm{CC}$ & 32.4 & 0.6 & 14.4 & 9.6 & - & - & 1.1 & - & 1.1 & 10.6 & - & 2.6 & - & - & - & - & - & - & 1.1 & 23.9 & 2.6 & - \\
\hline $8-2$ & 31.9 & 0.5 & 7.3 & 17.1 & 30.4 & - & - & 1.0 & - & - & - & - & - & - & - & - & 0.5 & - & - & 11.3 & - & - \\
\hline \multicolumn{23}{|l|}{ Site 325} \\
\hline $1-4$ & 34.7 & - & 17.8 & 12.6 & - & 0.5 & 1.0 & - & 0.5 & 2.6 & - & - & 1.0 & - & - & - & - & - & - & 29.3 & - & - \\
\hline & 32.9 & 0.5 & 8.5 & 14.1 & 1.4 & - & 0.9 & - & 0.9 & - & - & - & - & - & - & 2.3 & - & - & - & & - & - \\
\hline $2, \mathrm{CC}$ & 20.8 & - & 15.2 & 21.3 & - & 0.5 & 2.0 & - & - & - & - & 6.6 & - & - & 4.6 & - & - & 0.5 & - & 27.5 & 1.0 & - \\
\hline & 28.5 & - & 9.3 & 15.3 & 1.6 & 0.5 & 1.1 & - & - & - & - & 1.1 & - & - & - & 2.2 & - & - & - & 40.4 & - & - \\
\hline $3, \mathrm{CC}$ & 18.6 & - & 9.8 & 17.2 & - & - & - & - & - & - & - & 6.2 & - & - & - & 9.8 & - & - & 0.5 & 25.5 & - & - \\
\hline $4-1$ & 8.0 & - & 3.8 & 4.3 & - & - & - & 0.5 & 1.6 & - & - & - & - & - & 65.2 & 1.6 & - & - & 3.2 & 11 & - & - \\
\hline $4-1$ & 18.2 & - & 5.8 & 13.0 & 5.8 & - & - & - & 0.6 & - & - & - & - & - & - & 3.9 & 1.3 & - & 2.6 & 46.9 & 1.9 & - \\
\hline $4, C C$ & 21.8 & 0.6 & 16.0 & 6.3 & - & - & 1.1 & 1.7 & 2.6 & - & 1.1 & 3.4 & 1.7 & - & 13.7 & - & - & 1.1 & - & 28.9 & - & - \\
\hline $6-1$ & 20.4 & - & 15.4 & 18.5 & 1.9 & 0.7 & 1.1 & - & 0.7 & - & - & - & - & - & - & - & - & 0.7 & - & 38.1 & 1.1 & - \\
\hline $7-2$ & 15.5 & 0.5 & 4.8 & 10.6 & - & - & 0.5 & - & - & - & 0.5 & 14.0 & - & - & - & - & - & - & 0.5 & 52.6 & 0.5 & - \\
\hline $9, \mathrm{CC}$ & 13.8 & - & - & 16.8 & - & - & 1.5 & - & - & - & - & 3.6 & 9.7 & - & - & 2.0 & - & 0.5 & - & 52.1 & - & - \\
\hline $9-3$ & 10.1 & - & 5.4 & 6.0 & - & - & 2.4 & - & 0.6 & - & - & 57.7 & 7.1 & - & - & 1.2 & - & 0.6 & - & 8.9 & - & - \\
\hline 10 & 13.1 & - & 6.1 & 36.9 & 0.8 & - & 0.8 & 0.4 & - & - & - & 2.4 & 2.0 & - & - & 1.6 & - & - & - & 35.9 & - & - \\
\hline $10-3$ & 18.0 & 0.5 & 10.9 & 27.5 & 4.4 & - & 1.6 & - & - & - & - & - & 1.6 & - & - & - & - & - & - & 33.9 & - & - \\
\hline
\end{tabular}


TABLE 13

Distribution of Minerals in Heavy Fraction of Sediments (Elutriated)

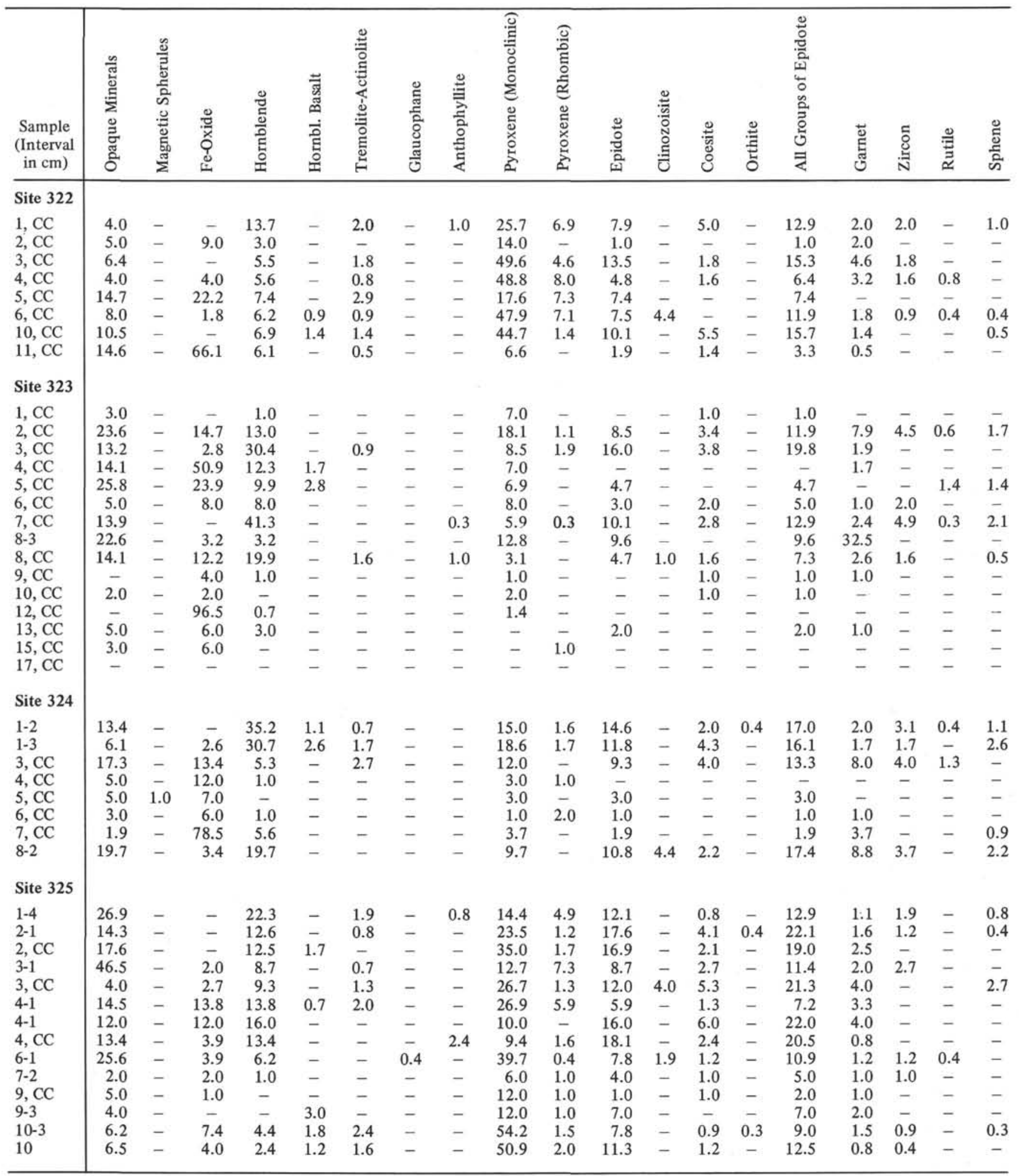




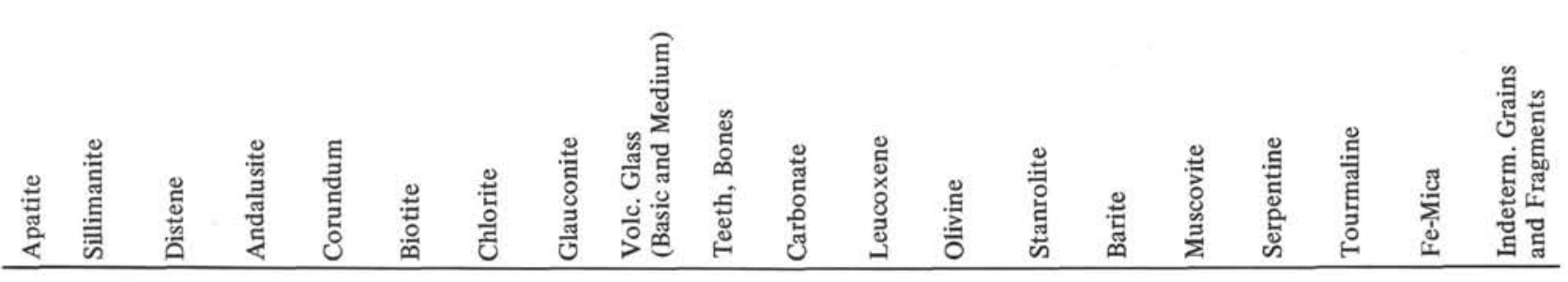

$\begin{array}{llllllllllllllllllll}2.0 & 1.0 & - & - & - & 4.0 & 3.0 & 1.0 & - & - & - & - & - & - & - & - & - & - & - & 17.8 \\ - & 1.0 & - & - & 1.0 & 2.0 & - & - & 2.0 & - & - & - & - & - & - & - & - & - & - & - \\ - & - & - & - & - & - & - & - & - & - & - & - & - & - & - & - & - & - & - & 10.4 \\ 0.8 & - & - & - & - & 0.8 & 1.6 & - & - & - & - & - & - & - & - & - & - & - & - & 13.6 \\ 2.9 & - & - & - & - & - & 2.9 & - & - & 5.9 & 8.8 & - & - & - & - & - & - & - & - & - \\ 1.8 & 0.4 & 0.4 & - & - & 0.4 & - & - & - & - & - & 0.4 & 0.9 & - & - & - & - & - & - & 7.5 \\ 1.4 & 0.5 & - & - & - & 0.9 & 1.4 & - & - & - & - & - & - & - & - & - & - & - & - & 11.0 \\ 1.4 & - & - & - & - & - & 0.9 & - & - & - & - & - & - & - & - & - & - & - & - & -\end{array}$

\begin{tabular}{|c|c|c|c|c|c|c|c|c|c|c|c|c|c|c|c|c|c|c|}
\hline- & - & - & - & - & - & - & - & - & - & - & - & - & - & - & - & - & 2.0 & - \\
\hline 0.6 & - & - & - & - & 0.6 & - & - & - & - & - & - & - & - & - & - & - & - & - \\
\hline 0.9 & 0.9 & - & - & 1.9 & 0.9 & - & - & - & - & - & 0.9 & - & - & - & - & - & - & 15.1 \\
\hline- & - & - & - & - & - & - & - & - & - & - & - & - & - & - & - & - & - & 12.3 \\
\hline- & - & - & - & 2.8 & - & - & - & - & 6.9 & - & - & - & - & - & - & - & - & 10.7 \\
\hline - & - & - & - & - & 1.0 & - & - & - & - & - & - & - & - & - & - & - & - & - \\
\hline 2.8 & 0.3 & - & - & - & - & - & 0.7 & - & - & 0.7 & 0.7 & - & - & - & - & - & - & 9.1 \\
\hline - & - & - & - & 3.2 & - & - & - & - & - & - & - & - & - & - & - & - & - & 12.9 \\
\hline - & - & - & - & 1.0 & 0.5 & - & - & - & 26.7 & - & 0.5 & - & - & - & - & - & - & 5.8 \\
\hline - & - & - & - & - & - & - & - & - & 2.0 & - & - & - & - & - & - & - & - & \\
\hline - & 1.0 & - & - & - & - & - & - & - & - & - & - & - & - & - & - & - & - & 5.0 \\
\hline - & - & - & - & - & - & - & - & - & - & - & - & - & - & - & - & - & - & 1.4 \\
\hline- & - & - & - & - & - & - & - & - & - & - & - & - & - & - & - & - & - & 2.0 \\
\hline - & - & - & - & - & - & - & - & - & 7.0 & - & - & - & - & - & - & - & - & - \\
\hline- & - & - & - & - & - & - & - & - & 100.0 & - & - & - & - & - & - & - & - & - \\
\hline
\end{tabular}

$\begin{array}{llllllllllllllllllll}5.0 & - & - & - & - & 1.6 & - & - & - & - & - & - & - & 0.4 & 0.4 & - & - & - & - & 2.0 \\ 1.7 & 1.7 & - & - & - & - & 1.7 & - & - & - & - & - & - & - & - & - & - & - & - & 8.8 \\ 2.7 & - & - & - & 2.7 & - & - & - & - & - & - & - & - & - & - & - & - & - & - & 17.3 \\ - & - & - & - & - & - & - & - & 1.0 & - & - & - & - & - & - & - & - & - & - & 5.0 \\ - & - & - & - & - & - & 1.0 & - & - & - & - & - & - & - & - & - & - & - & - & - \\ - & - & - & - & - & - & - & - & - & - & - & - & - & - & - & - & - & - & - & 4.0 \\ 1.9 & - & - & - & - & - & 1.9 & - & - & - & - & - & - & - & - & - & - & - & - & - \\ 1.5 & - & - & 0.7 & - & - & 1.5 & - & - & - & 4.4 & - & 1.5 & - & - & 0.7 & - & - & 5.1 & -\end{array}$

$\begin{array}{rllllllllllllllllllll}1.5 & - & 0.4 & - & - & 0.4 & - & - & - & - & 0.8 & - & - & - & - & - & - & - & - & 9.0 \\ 0.8 & 0.8 & - & - & - & 1.2 & 0.8 & - & - & - & 0.4 & 0.4 & - & - & - & 0.8 & - & - & - & 17.1 \\ 0.8 & - & - & - & - & - & 1.7 & - & - & - & - & - & - & - & - & - & - & - & - & 7.5 \\ 1.3 & 0.7 & - & - & - & - & - & - & - & - & - & - & 0.7 & - & - & - & - & - & - & 3.3 \\ - & - & - & - & - & - & - & - & - & - & 20.0 & - & 4.0 & - & - & - & - & - & - & 2.7 \\ 0.7 & - & - & - & - & - & - & - & - & - & - & - & - & - & - & - & - & - & - & 11.2 \\ - & - & - & - & - & - & 4.0 & - & 4.0 & - & - & - & 4.0 & - & - & - & 2.0 & - & - & 10.0 \\ 0.8 & - & - & - & - & 1.6 & 0.8 & - & - & - & - & 0.8 & 0.8 & - & - & - & - & 0.8 & - & 29.0 \\ - & - & - & - & - & - & - & - & - & - & - & - & - & - & - & - & 0.4 & - & - & 9.7 \\ - & - & - & - & - & - & - & - & - & - & - & - & - & - & - & - & - & - & - & 4.0 \\ - & - & - & - & - & - & - & - & - & - & 1.0 & - & - & - & - & - & - & - & - & 9.0 \\ - & - & - & - & - & - & 1.0 & - & - & - & - & 1.0 & - & - & - & - & - & - & - & 9.0 \\ - & - & - & 0.3 & - & 0.9 & 0.9 & 0.3 & - & - & - & 0.6 & - & 0.3 & - & - & - & - & - & 7.1 \\ 1.6 & - & 0.8 & - & - & 0.4 & 0.8 & - & - & - & - & 0.4 & - & 0.4 & - & - & - & - & 1.6 & 11.7\end{array}$


TABLE 14

X-Ray Mineralogy (All Sample)

\begin{tabular}{|c|c|c|c|c|c|}
\hline $\begin{array}{c}\text { Sample } \\
\text { (Interval in } \mathrm{cm} \text { ) }\end{array}$ & 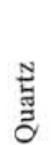 & 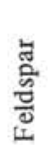 & 䒕 & 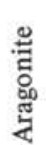 & 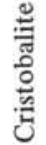 \\
\hline
\end{tabular}

Site 322

1-2, 26-30

$1-3,70-76$

3-1, 18-20

4-1, $65-67$

4-2, 57-60

5-1, 81-85

6-1, 30-33

9-2, 143-146

10-1, 101-105

10-2, 43-46

$11-2,45-49$

11-3, 24-32

$11-4,17-24$

$11-6,109-115$

$\begin{array}{rrrrr}23 & 22 & - & - & - \\ 10 & 12 & - & - & - \\ 11 & 13 & - & - & - \\ 6 & 7 & - & - & - \\ 9 & 15 & - & - & - \\ 8 & 13 & - & - & - \\ 10 & 11 & - & - & - \\ 7 & 16 & - & - & - \\ 8 & 11 & - & - & - \\ 10 & 17 & - & - & - \\ 10 & 18 & - & - & - \\ 15 & 20 & - & - & - \\ 13 & 25 & - & - & - \\ 18 & 7 & - & - & -\end{array}$

Site 323

\begin{tabular}{lrrlll}
$1-1,50-60$ & 55 & 30 & - & - & - \\
$1-1,140-145$ & 27 & 15 & - & - & - \\
$1-2,34-43$ & 9 & 8 & - & - & - \\
$1-2,78-88$ & 12 & 11 & - & - & - \\
$1-4,118-127$ & 12 & 8 & - & - & - \\
$3-1,88-94$ & 15 & 12 & - & - & - \\
$3-2,14-20$ & 35 & 22 & - & - & - \\
$3-2,61-70$ & 12 & 18 & - & - & - \\
$7-2,105-110$ & 8 & 12 & - & - & - \\
$7-3,18-24$ & 11 & 8 & - & - & - \\
$8-1,120-121$ & 6 & 4 & - & - & 60 \\
$10-1,113-122$ & 17 & 22 & - & - & - \\
$10-2,75-85$ & 10 & 9 & - & - & - \\
$10-3,106-114$ & 18 & 12 & - & - & - \\
$11-1,22-32$ & 20 & 10 & - & - & - \\
$11-2,137-148$ & 24 & 15 & - & - & - \\
$13-5,106-115$ & 20 & 10 & - & - & - \\
$13-6,145-150$ & 27 & 10 & - & - & - \\
$14-2,1-8$ & 26 & 9 & - & - & - \\
$14-2,128-135$ & 20 & 8 & - & - & - \\
$15-1,52-60$ & 12 & 4 & - & - & - \\
$15-2,91-100$ & 18 & 8 & - & - & - \\
$15-3,29-36$ & 12 & 6 & - & - & - \\
$15-4,54-63$ & 17 & 4 & - & - & - \\
$15-5,89-98$ & 7 & 4 & - & - & - \\
$15-6,19-26$ & 12 & 3 & 17 & 2 & - \\
$16-1,57-62$ & 12 & 3 & 40 & - & - \\
$16-2,30$ & 12 & 2 & - & - & - \\
$16-2,58$ & 7 & 2 & - & - & - \\
$16-3,32-37$ & 10 & 1 & - & - & - \\
$16-4,83-94$ & 6 & 6 & - & - & - \\
$18-2,80-86$ & 10 & 6 & - & - & - \\
$18-3,130-139$ & 10 & 6 & - & - & - \\
\hline & & & & &
\end{tabular}

TABLE 14 - Continued

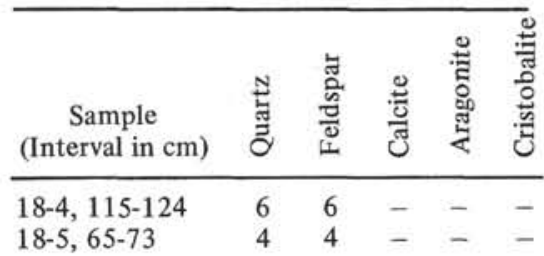

\section{Site 324}

$\begin{array}{lrrrrr}1-3,124-132 & 20 & 14 & - & - & - \\ 1-3,126-134 & 12 & 12 & - & - & - \\ 1-4,125-131 & 20 & 15 & - & - & - \\ 1-6,36-38 & 22 & 30 & - & - & - \\ 2-1,83-92 & 18 & 12 & - & - & - \\ 2-2,61-70 & 20 & 8 & - & - & - \\ 2-3,52-61 & 24 & 14 & - & - & - \\ 2-4,27-36 & 25 & 14 & - & - & - \\ 2-5,35-45 & 20 & 13 & - & - & - \\ 2-6,95-104 & 23 & 13 & - & - & - \\ 3-1,137-146 & 27 & 14 & - & - & - \\ 3-2,136-145 & 22 & 12 & - & - & - \\ 3-3,67-77 & 25 & 12 & - & - & - \\ 3-4,47-57 & 23 & 10 & - & - & - \\ 3-5,99-110 & 21 & 7 & - & - & - \\ 3-6,138-148 & 20 & 19 & - & - & - \\ 4-2,113-123 & 18 & 11 & - & - & - \\ 4-3,30-40 & 16 & 12 & - & - & - \\ 4-4,54-64 & 20 & 12 & - & - & - \\ 5-2,66-83 & 23 & 14 & - & - & - \\ 5-3,25-35 & 30 & 14 & - & - & - \\ 6-2,19-30 & 24 & 11 & - & - & - \\ 7-1,69-78 & 30 & 11 & - & - & - \\ 7-3,110-121 & 25 & 12 & - & - & - \\ 7-6,119-129 & 28 & 12 & - & - & - \\ 8-3,61-71 & 25 & 9 & - & - & -\end{array}$

\section{Site 325}

\begin{tabular}{lrrrrr}
$1-1,49-59$ & 11 & 10 & - & - & - \\
$1-1,133-144$ & 9 & 12 & 1 & - & - \\
$1-2,26-36$ & 8 & 12 & - & - & - \\
$1-4,81-92$ & 16 & 18 & - & - & - \\
$2-2,39-49$ & 9 & 16 & - & - & - \\
$3-2,11-24$ & 7 & 10 & - & - & - \\
$3-3,76-87$ & 13 & 20 & - & - & - \\
$3-4,26-36$ & 10 & 17 & - & - & - \\
$4-2,90$ & 13 & 17 & - & - & - \\
$4-3,20-26$ & 12 & 15 & - & - & - \\
$4-3,130$ & 15 & 13 & - & - & - \\
$4, C C$ & 20 & 20 & - & - & - \\
$5-1,55-57$ & 10 & 14 & - & - & - \\
$8-1,45-49$ & 10 & 9 & - & - & - \\
$8-2,119-128$ & 11 & 9 & 6 & - & - \\
$8-3,27-35$ & 9 & 12 & - & - & - \\
$9-3,90-98$ & 18 & 19 & - & - & - \\
\hline
\end{tabular}


TABLE 15

Characteristic Mineral Complexes of Leg 35 Sediments

\begin{tabular}{|c|c|c|c|c|c|c|}
\hline \multirow[b]{2}{*}{ Minerals } & \multicolumn{2}{|c|}{ Complex 1} & \multicolumn{2}{|c|}{ Complex 2} & \multicolumn{2}{|c|}{ Complex 3} \\
\hline & Range & Average & Range & Average & Range & Average \\
\hline \multicolumn{7}{|c|}{ Heavy Minerals } \\
\hline $\begin{array}{l}\text { Monoclinic } \\
\text { Pyroxene }\end{array}$ & $1.53-53.3$ & 39.2 & $1.6-26.0$ & 10.4 & $0-7.2$ & 0.1 \\
\hline $\begin{array}{l}\text { Rhombic } \\
\text { Pyroxene }\end{array}$ & $0-15.5$ & 2.0 & $0-4.7$ & 1.3 & $0-0.3$ & 0.1 \\
\hline Epidote & $0-35.4$ & 18.0 & $9.7-32.5$ & 23.3 & $0-9.3$ & 3.1 \\
\hline Hornblende & $0.0-7.9$ & 3.3 & $12.8-33.8$ & 25.1 & $0-2.1$ & 0.6 \\
\hline $\begin{array}{l}\text { Opaque } \\
\text { Minerals }\end{array}$ & $3.0-43.4$ & 12.6 & $8.3-31.2$ & 15.4 & $0-90.0$ & 14.8 \\
\hline Fe-oxide & $0-49.0$ & 2.2 & $0.22-16.7$ & 2.8 & $2.9-98.4$ & 47.6 \\
\hline $\begin{array}{l}\text { Pyrite- } \\
\text { Marcasite }\end{array}$ & $0-35.0$ & 5.3 & $0-7.9$ & 2.2 & $0-28.8$ & 4.2 \\
\hline Garnet & $0-12.5$ & 1.7 & $0-8.4$ & 2.6 & $0-0.5$ & 0.2 \\
\hline Apatite & $0-0.6$ & 0.2 & $0.21-4.8$ & 1.6 & $0-0.7$ & 0.1 \\
\hline Zircon & $0-2.3$ & 0.4 & $0-5.7$ & 1.8 & $0-0.5$ & 0.2 \\
\hline Sphene & $0-1.0$ & 0.2 & $0-3.9$ & 1.1 & $0-0.2$ & 0.1 \\
\hline Barite & $0-1.7$ & 0.1 & $0-0.7$ & 0.1 & $0-73.8$ & 9.8 \\
\hline $\begin{array}{l}\text { Indeterm. } \\
\text { Minerals } \\
\text { and } \\
\text { Fragments }\end{array}$ & $1.4-18.5$ & 9.1 & $0-11.5$ & 5.3 & $0-3.7$ & 0.9 \\
\hline \multicolumn{7}{|c|}{ Light Minerals } \\
\hline Quartz & $4.6-39.4$ & 15.1 & $7.1-48.5$ & 26.6 & $0-47.5$ & 9.1 \\
\hline Feldspar & $1.2-11.7$ & 5.3 & $0.5-26.0$ & 12.9 & $0-23.1$ & 3.5 \\
\hline $\begin{array}{l}\text { Plagioclase } \\
\text { Acid }\end{array}$ & $0-12.3$ & 2.8 & $0.3-6.4$ & 2.6 & $0-7.1$ & 1.2 \\
\hline $\begin{array}{l}\text { Plag. Basic } \\
\text { and Medium }\end{array}$ & $5.5-37.0$ & 20.2 & $0-13.0$ & 2.8 & $0-8.3$ & 1.5 \\
\hline $\begin{array}{l}\text { Volc. Glass } \\
\text { Acid }\end{array}$ & $0-3.7$ & 0.5 & $0-36.5$ & 3.1 & $0.4-35.0$ & 6.7 \\
\hline $\begin{array}{l}\text { Volc. Glass } \\
\text { Basic }\end{array}$ & $0-3.4$ & 0.3 & $0-1.0$ & 0.1 & 0.0 & 0.0 \\
\hline Volc. Ash & $0-28.4$ & 5.5 & $0-17.2$ & 1.4 & $0-1.4$ & 0.2 \\
\hline Zeolite & $0-23.4$ & 1.9 & $0-8.6$ & 0.4 & $0-81.0$ & 10.8 \\
\hline $\begin{array}{l}\text { Teeth, } \\
\text { Bones }\end{array}$ & $0-8.5$ & 0.3 & $0-4.3$ & 0.5 & $0-34.0$ & 15.1 \\
\hline $\begin{array}{l}\text { Clay } \\
\text { Fragments }\end{array}$ & $0-57.6$ & 14.5 & $0-76.9$ & 31.5 & $0-45.4$ & 23.6 \\
\hline $\begin{array}{l}\text { Indeterm. } \\
\text { Minerals } \\
\text { and } \\
\text { Fragments }\end{array}$ & $0-53.0$ & 25.6 & $0-39.5$ & 14.2 & $0-24.6$ & 4.3 \\
\hline
\end{tabular}

TABLE 16

Petrography of Gravel of Site 323, Core 4, and Site 255, Core 2, Section 1

\begin{tabular}{lc}
\hline \multicolumn{1}{c}{ Rock } & Frequency of Occurrence \\
\hline Diabase & ++ \\
Quartz-Feldspar & ++ \\
Pegmatoid Granite & + \\
Aplitic Granite & + \\
Gabbro & ++ \\
Siliceous Rock & +++ \\
Siliceous-carbonaceous Slate & + \\
Sedimentary rock fragments & ++ \\
Tuff & ++ \\
Epidosite & + \\
Gneiss & + \\
Quartz-feldspar Slate & ++ \\
\hline
\end{tabular}

